\title{
RESOURCE MANAGEMENT AND
} NETWORK LAYER

Editors: Ulla Birnbacher ${ }^{1}$, Wei Koong Chai ${ }^{2}$

Contributors: Paolo Barsocchi ${ }^{3}$, Ulla Birnbacher ${ }^{1}$, Wei Koong Chai ${ }^{2}$, Antonio Cuevas ${ }^{4}$, Franco Davoli ${ }^{5}$, Alberto Gotta ${ }^{3}$, Vincenzo Mancuso ${ }^{6}$, Mario Marchese $^{5}$, Maurizio Mongelli ${ }^{5}$, José Ignacio Moreno Novella ${ }^{4}$, Francesco Potorti $^{3}$, Orestis Tsigkas ${ }^{7}$

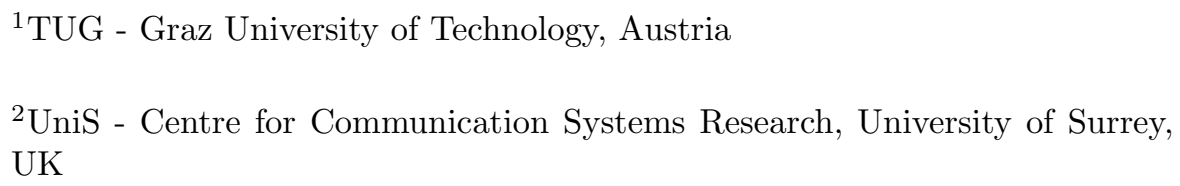

\subsection{Introduction}

The Internet protocols have become the worldwide standard for network and transport protocols and are increasingly used in satellite communication 
networks. Also traditional telecommunication and broadcast applications like VoIP and video streaming are transported over the Internet, although it does not support natively applications with tight QoS requirements. In satellite communication networks, further challenges arise, as bandwidth resources are limited and physical transmission time adds some more pressure on delay constraints. Since resources are limited, the efficient assignment of bandwidth to different data streams has always been an issue for satellite communications. However, supporting QoS for IP-based applications results in additional requirements for resource allocation. In order to provide QoS for applications, several layers of the protocol stack of a satellite communication system will need to be adapted or have to interact with each other in some way. This Chapter will concentrate on different resource management schemes at the MAC layer (layer 2) for supporting IP QoS (layer 3).

This Chapter begins with an overview of the current IP QoS frameworks in Section 8.2. In Section 8.3, the discussion is focused on the interaction of layer 2 and layer 3 in satellite environments for the support of IP QoS. This Section ends with an example of implementation for a variant of one of the most popular IP QoS frameworks. The following Section 8.4 provides an in-depth work on achieving QoS requirements by a cross-layer approach over SI-SAP. Section 8.5 looks into another aspect of resource management: the QoS provisioning for terminals supporting dual network access (WiFi and satellite). Implicit cross-layer design methodology is used in Section 8.6 for switched Ethernet over LEO satellite networks. Finally, this Chapter is concluded in Section 8.7. In the studies carried out in this Chapter, Scenario 2 (i.e., GEO-based DVB-S/-RCS systems; see Chapter 1, Section 1.4) has been adopted, except for the considerations made in Section 8.6, where Scenario 3 (i.e., LEO satellite) has been considered.

\subsection{Overview IP QoS framework}

In order to support the emerging Internet QoS, some QoS frameworks have been proposed. These service models and mechanisms evolve the IP architecture to support new service definitions that allow preferential or differentiated treatment to be provided to certain traffic types. Integrated Services and Differentiated Services have already been introduced in Section 3.3, but are discussed below in more detail with satellite networks in mind, including Multiprotocol Label Switching (MPLS).

\subsubsection{Integrated services}

The Integrated Services (IntServ) model [1] requires resources, such as bandwidth and buffers, to be reserved a priori for a given traffic flow to ensure that the QoS requested by this traffic flow is fulfilled. The IntServ model includes additional components beyond those used in the best-effort model 
such as packet classifiers, packet schedulers, admission control and signaling. A packet classifier is used to identify flows that have to receive a certain level of service. A packet scheduler manages the service provided to different packet flows to ensure that QoS commitments are met. Admission control is used to determine whether a router has the necessary resources to accept a new flow.

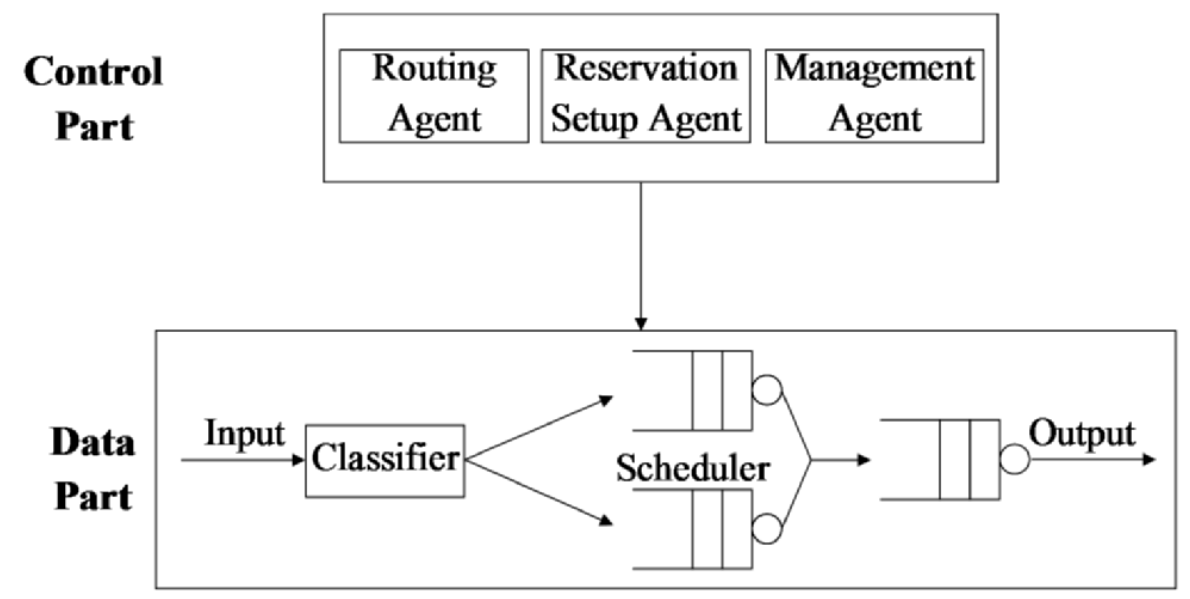

Fig. 8.1: Implementation reference model for routers with IntServ [2].

A notable feature of the IntServ model is that it requires explicit signaling of QoS requirements from end-systems to routers. The Resource Reservation Protocol (RSVP) [3] performs this signaling function and is a critical component of IntServ. RSVP is a soft state signaling protocol. It supports receiver-initiated establishment of resource reservations for both multicast and unicast flows. Recently, RSVP has been modified and extended in several ways to reserve resources for aggregation of flows, to set up MPLS explicit label switched paths with QoS requirements, and to perform other signaling functions within the Internet.

Two services have been defined under the IntServ model: guaranteed service [4] and controlled-load service [5]. The guaranteed service provides a firm quantitative bound on the end-to-end packet delay for a flow. This is accomplished by controlling the queuing delay on network elements along the data flow path. The guaranteed service model does not, however, provide bounds on jitter (inter-arrival times between consecutive packets). The controlled-load service can be used for adaptive applications that can tolerate some delay, but are sensitive to traffic overload conditions. This type of application typically operates satisfactorily when the network is lightly loaded, but its performance degrades significantly when the network is heavily loaded. Controlled-load service, therefore, has been designed to provide approximately 
the same service as best-effort service in a lightly loaded network regardless of actual network conditions. Controlled-load service is described qualitatively in that no target values of delay or loss are specified.

The IntServ architecture represents a fundamental change to the current Internet architecture, which is based on the concept that all flow-related state information should be in the end-systems. The main problem of the IntServ model is scalability, especially in large public IP networks, which may potentially have millions of active micro-flows concurrently in transit, since the amount of state information maintained by network elements tends to increase linearly with the number of micro-flows.

\subsubsection{Differentiated services}

One of the primary motivations for Differentiated Services (DiffServ) [6] was to devise alternative mechanisms for service differentiation in the Internet that mitigate the scalability issues encountered with the IntServ model. Scalable mechanisms are deployed within the DiffServ framework for the categorization of traffic flows into behavior aggregates, allowing each behavior aggregate to be treated differently, especially when there is shortage of resources such as link bandwidth and buffer space.

A DiffServ field in the IPv4 header has been defined. Such field consists of six bits of the part of the IP header, formerly known as TOS octet, and it is used to indicate the forwarding treatment that a packet should receive at a node. Within the DiffServ framework, a number of Per-Hop Behavior (PHB) groups have been also standardized. Using the PHBs, several classes of services can be defined using different classification, policing, shaping, and scheduling rules.

Conceptually, a DiffServ domain consists of two types of routers, namely core router and edge router. Core router resides within the domain and is generally in charge of forwarding packets based on their respective DiffServ Code Point (DSCP). The edge router is located at the boundary of the network domain which will either further connect to another domain (inter-domain) or to end-users. It can be further categorized as ingress router which operates on traffic flowing into the domain and egress router which operates on traffic exiting the domain.

In order for an end-user to receive DiffServ from its Internet Service Provider (ISP), it may be necessary for the user to have a Service Level Agreement (SLA) with the ISP. An SLA may explicitly or implicitly specify a Traffic Conditioning Agreement (TCA), which defines classifier rules, as well as metering, marking, discarding, and shaping rules. Packets are classified, and possibly policed and shaped at the ingress routers of a DiffServ network according to SLAs.

When a packet traverses the boundary between different DiffServ domains, the DiffServ field of the packet may be re-marked according to existing agreements between the domains. DiffServ allows only a finite number of 


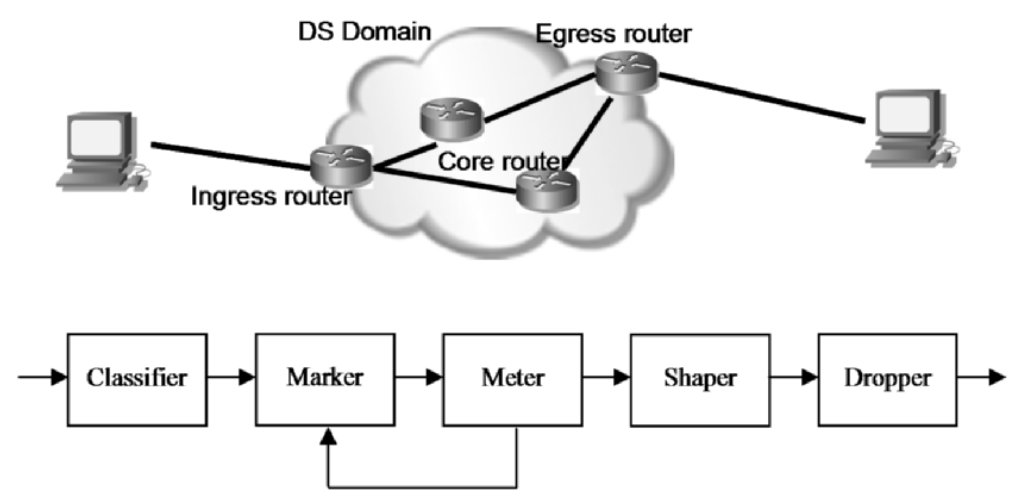

Fig. 8.2: DiffServ network; (top) DiffServ domain illustration; (bottom) logical view of DiffServ packet classifier and traffic conditioner.

service classes to be indicated by the DiffServ field.

The main advantage of the DiffServ approach relative to the IntServ model is scalability. Resources are allocated on a per-class basis and the amount of state information in the routers is proportional to the number of classes rather than to the number of application flows. A second advantage of the DiffServ approach is that sophisticated classification, marking, policing, and shaping operations are only needed at the boundary of the network.

The DiffServ control model essentially deals with traffic management issues on a per-hop basis and consists of a collection of micro-control mechanisms. Other traffic engineering capabilities, such as capacity management (including routing control), are also required in order to deliver acceptable QoS in DiffServ networks.

At the current stage, the DiffServ approach is still evolving. Two directions of its development can be categorized: namely, absolute DiffServ and relative DiffServ [7]. The absolute DiffServ approach is the more traditional approach detailed above. The newer and simpler approach is the relative DiffServ, whereby QoS assurances are provided relative to the ordering between several traffic or service classes rather than specifying the actual service level or quality of each class. This approach is lightweight in nature, since it minimizes computational cost as it does not require sophisticated mechanisms such as admission control and resource reservation. As such, recently, it has gain in popularity not only in terrestrial networks, but also in wireless $[8],[9]$ and satellite systems [10].

\subsubsection{Multiprotocol Label Switching (MPLS)}

MPLS is an advanced forwarding scheme, which extends the Internet routing model and enhances packet forwarding and path control [11]. MPLS stands for Multiprotocol Label Switching; note that the word 'multiprotocol' is used, 
because these techniques are applicable to any network layer protocol.

In conventional IP forwarding, when a packet of a connectionless network layer protocol travels from one router to the next, each router makes an independent forwarding decision for that packet. That is, each router re-examines the packet's header and independently chooses a next hop for the packet, based on the results of the routing algorithm. Choosing the next hop can be thought of as the composition of two functions. The first function partitions the entire set of possible packets into a set of Forwarding Equivalence Classes; the second function maps each class to a next hop.

In the MPLS forwarding paradigm, the assignment of a particular packet to a given class is done just once, at the ingress to an MPLS domain, by Label Switching Routers (LSRs). As the forwarding decision is concerned, different packets that get mapped into the same class are indistinguishable and will follow the same path. The class to which the packet is assigned is encoded as a short fixed-length value known as a label. When a packet is forwarded to its next hop, the label is sent along with it; that is, packets are labeled before they are forwarded. At subsequent hops, there is no further analysis of the packet network layer header. Rather, the label is used as an index into a table, which specifies the next hop, and a new label. The old label is replaced by the new label, and the packet is forwarded to its next hop. Most commonly, a packet is assigned to a class based (completely or partially) on its destination IP address. However, the label never is an encoding of that address.

A Label Switched Path (LSP) is the path between an ingress LSR and an egress LSR through which a labeled packet traverses. The path of an explicit LSP is defined at the originating (ingress) node of the LSP. MPLS can use a signaling protocol such as RSVP or Label Distribution Protocol (LDP) to set up LSPs. MPLS is a very powerful technology for Internet traffic engineering because it supports explicit LSPs, which allow constraint-based routing to be implemented efficiently in IP networks.

\subsection{Resource management for IP QoS}

Resource management schemes at MAC layer (layer 2) are essential in supporting IP QoS (layer 3). The current IP QoS frameworks (i.e., IntServ and DiffServ) define several service classes to cater for users with different QoS requirements. The resource management scheme must be able to allocate dynamically the available resources in an IP-based satellite network to achieve the requirements of the defined service classes. This includes a mapping scheme between layer 3 and layer 2, dynamic bandwidth allocation and scheduling mechanisms.

In this Section, the specific scenario under consideration is a DiffServ satellite domain with DVB-RCS architecture for multimedia fixed unicast users. The choice of DiffServ is mainly in view of the problems of the IntServ framework, such as scalability and deployment. For DiffServ, in general, there 
are three types of PHBs being used: namely Expedited Forwarding (EF), Assured Forwarding (AF) and Best Effort (BE). EF PHB caters for low loss, low delay and low jitter services. The AF PHB consists of four AF classes, where each class is allocated with different amounts of buffer and bandwidth. Hence, each subscriber with a specific Subscribed Information Rate will receive assured performance for traffic within such rate while excess traffic may be lost depending on the current load of the AF class. Finally, the BE PHB is the same as the original best effort IP paradigm.

For the DVB-RCS architecture, there are four transmission capacity allocation schemes; namely Continuous Rate Assignment (CRA), Rate Based Dynamic Capacity (RBDC), Volume Based Dynamic Capacity (VBDC) and

Free Capacity Assignment (FCA). For the description of these different resource allocation schemes, please refer to Chapter 1, sub-Section 1.4.3.

Before mapping the DiffServ PHBs to DVB-RCS resource allocation schemes, it is vital to note that the entire DiffServ domain is assumed to be properly dimensioned. This is because there is no one mapping scheme that can achieve high efficiency in all types of traffic mixture. A particular scheme, which performs well in one scenario, may perform poorly in another. The network management and dimensioning problem is not within the scope of this study.

Usually, EF PHB is used to transport non-delay tolerant application traffics such as VoIP and video conferencing. To achieve the stringent QoS requirements of this class of applications, the use of CRA in the MAC layer is a must. However, considering system efficiency, a minimal use of RBDC combined with CRA is plausible. The entire DiffServ domain has to be properly dimensioned as noted above. For example, if a very high traffic percentage is of the EF type, then the satellite bandwidth will be quickly consumed with all the slots being reserved with CRA, thus causing high blocking and drop rate. As for AF PHB, the combined use of RBDC and VBDC is proposed with RBDC as the main resource provider. Under low load, packets belonging to each class of $\mathrm{AF}$ will receive similar treatment. However, to differentiate between the $\mathrm{AF}$ classes, a different maximum RBDC value (i.e., maximum bit-rate that can be allocated with RBDC) can be defined so that the higher $\mathrm{AF}$ class will receive better treatment. If the request is higher than the maximum RBDC, the users can still request for VBDC resources. For BE traffic, the use of VBDC and FCA is proposed.

\subsubsection{Relative DiffServ by MAC Scheduling}

An alternative scenario on resource management schemes at MAC layer (layer 2) to support IP QoS (layer 3) is the work on attempting to realize relative service differentiation in a Bandwidth on Demand (BoD) satellite IP network. The Proportional Differentiated Service (PDS) [7] model is one of the most recent developments of DiffServ in the direction of relative service differentiation. It strives to strike a balance between the strict QoS guarantee 
of IntServ and the scalability of DiffServ. Similarly to DiffServ, PDS segregates traffics into a finite number of service classes. However, it does not provide them with absolute QoS guarantees. Instead, it controls the performance gap between each pair of service classes, i.e., quantitative relative differentiation amongst the supported classes.

Formally, the PDS model requires

$$
\frac{\sigma_{i}}{\sigma_{j}}=\frac{r_{i}}{r_{j}} ; \forall i, j \in\{1 \ldots N\} .
$$

where each class is associated with a Differentiation Parameter (DP), $r_{i}$, and $\sigma_{i}$ is the performance metric of interest for class $i$, e.g., throughput, packet loss or queuing delay. $N$ is the total number of supported service classes in the network.

In this Section, classes are numbered in decreasing priority order, i.e., the lower the class index, the better the service provided to it. All DPs are normalized with reference to the highest priority class $(=1)$ :

$$
0<r_{N}<r_{N-1}<\ldots<r_{2}<r_{1}=1
$$

This Section demonstrates how such a model can be realized in an IP-based broadband multimedia BoD GEO satellite network with resource allocation mechanisms analogous to the DVB-RCS system standard [12].

Figure $8.3[10]$ illustrates the main nodes of the network architecture:

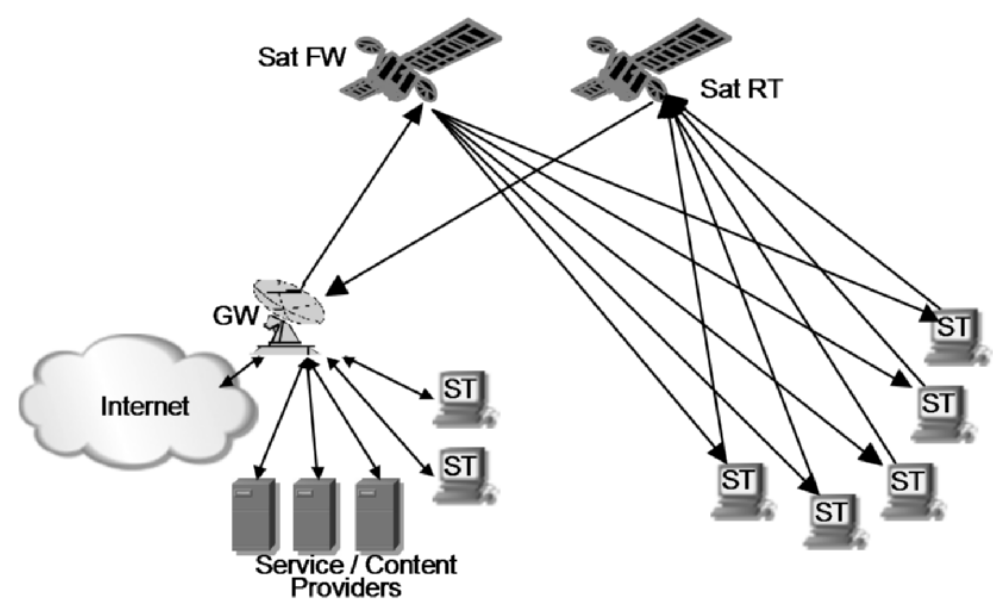

Fig. 8.3: Reference satellite system, resembling the DVB-RCS architecture. See reference [10]. Copyright (C)2005 IEEE. 
- Satellite(s): The satellite is assumed to be equipped with On-Board Processor (OBP) and the scheduler is located on-board.

- Traffic Gateway (GW): In line with the DVB-RCS definition, GWs are included to provide interactive services to networks (e.g., Internet) and service providers (e.g., databases, interactive games, etc.).

- Satellite Terminal (ST): STs represent the users. They may represent one (residential) or more users (collective).

Time Division Multiple Access (TDMA) is used for the forward path whereas on the return path, Multi Frequency - TDMA (MF-TDMA) is assumed. In an MF-TDMA frame, the basic unit of the link capacity is the Time Slot (TS) with multiple TSs grouped in TDMA frames along several frequency carriers. In this Section, fixed MF-TDMA frame is considered whereby the bandwidth and duration of successive TSs is static. For more details on MF-TDMA characteristics, please refer to Chapter 1.

The BoD scheme used in this Section is derived from [13]. It is a cyclic procedure between two stages: the resource request estimation stage and the resource allocation stage. It involves the $\mathrm{BoD}$ entity located at the ST and $\mathrm{BoD}$ scheduler located onboard the satellite. The BoD entity handles all packets of the same class which are stored in the same queue, i.e., there will be $x \mathrm{BoD}$ entities in an $\mathrm{ST}$ if this ST supports $x$ classes. In the resource request estimation stage, the $\mathrm{BoD}$ entities (i.e., STs) periodically compute and send Slot Requests (SRs) to the BoD scheduler, when there are new packet arrivals at their queues. In the resource allocation stage, upon reception of SRs, the BoD scheduler allocates TSs to each requesting BoD entity based on a certain scheduling discipline and policies defined by the network operator. It then constructs and broadcasts the Terminal Burst Time Plan (TBTP) that contains all the resource allocation information to the BoD entities. Figure 8.4 [10] gives the BoD timing diagram, which also describes the basic tasks involved.

Due to the unique characteristics of satellite networks, the realization of such framework is very different from those solutions provided for terrestrial and wireless systems. For terrestrial wired networks, the scheduler only needs to schedule the departure of each contending packet locally within a router. In wireless and satellite domain, the access to the transmission medium is often controlled in a distributed manner by a MAC protocol. Hence, packets from one node may contend with packets from other nodes. This leads to the consideration of using layer 2 scheduling to realize the model instead of purely depending on layer 3. Based on the layer 3 QoS classes, the MAC layer scheduler will decide how best to schedule the packets in order to achieve the QoS required.

Moreover, there are several fundamental architectural and environmental differences between terrestrial wireless networks and satellite networks supporting dynamic bandwidth allocation mechanisms. Firstly, for a BoD-based satellite architecture, resource has to be requested by the STs before they can 


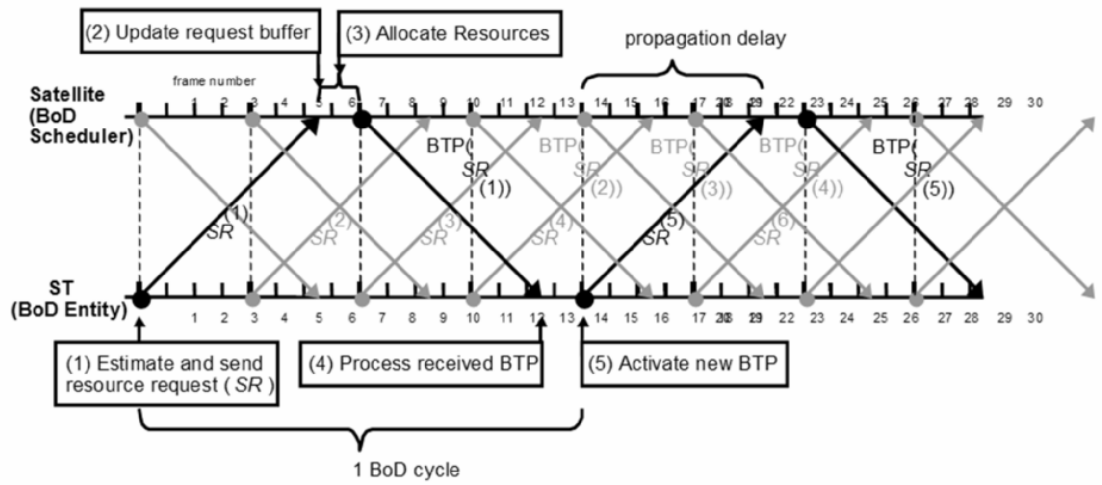

Fig. 8.4: BoD timing diagram. See reference [10]. Copyright (C)2005 IEEE.

make use of it, so that the scheduler ends up scheduling requests for resource rather than packets. Secondly, there is a non-negligible propagation delay between the STs and the scheduler that may, depending on the access control algorithm, inflate the waiting time of a packet in the ST queue. The impact of this semi-constant delay has to be taken into account by the scheduler in providing relative service differentiation.

The Satellite Waiting Time Priority (SWTP) scheduler is a satellite adaptation of the Waiting Time Priority scheduler [7], proposed by Kleinrock in [14]. SWTP schedules SRs from BoD entities rather than individual packets. SWTP has been shown to be able to provide proportional queuing delay to several classes of MAC frames in the context of BoD environment. Its main elements are as follow.

1. Resource request. Formally, if $Q_{i}^{m}$ is the set of newly arrived packets at the $i$-th queue of $\mathrm{BoD}$ entity $m$, i.e., packets that came within the last resource allocation period, $q$ the set cardinality, and $\tau_{j}$ the arrival time of packet $j, 1 \leq j \leq q$, indexed in increasing order of arrival times, then the BoD entity $m$ computes at time $t$ the SR timestamp $t s_{i}^{m}$, according to the arrival time of the last packet that arrived in the queue during the last resource allocation period, namely: $t s_{i}^{m}=t-\tau_{q}$.

2. Resource allocation: the BoD scheduler computes the priority of each SR. The priority $P_{i}^{m}(k)$, assigned to $S R_{i}^{m}$ in the $k$-th resource allocation period is

$$
P_{i}^{m}(k)=r_{i} \cdot\left(w_{i}^{S R}(k)+\alpha\right)
$$

where $\alpha$ accounts for the propagation delay of TBTP and the processing delay of BoD entities, while $w_{i}^{S R}(k)=t-t s_{i}^{m}$ and $t s_{i}^{m}$ is the timestamp information encoded in each SR. Finally, $r_{i}$ denotes here the Delay 
Differentiation Parameter (DDP): each one of the $N$ MAC classes is attached with a specific $r_{i}, 1 \leq i \leq N$. At each allocation period, the SWTP allocates TSs by considering requests in decreasing priority order: requests are fully satisfied as long as they do not exceed the available capacity. All unsatisfied requests will be buffered for the next allocation period. At the next allocation period, the priorities of the buffered SRs will be recalculated to account for the additional waiting time of SRs at the scheduler.

The setup of the simulations is as follow. The capacities for all links are configured to be $2048 \mathrm{kbit} / \mathrm{s}$. Unless explicitly stated otherwise, the network is set to have DDPs: $1,1 / 2,1 / 4,1 / 8$. By this setting where the differentiation is exactly half of the next adjacent class, the ideal performance ratio according to the PDS model will be 0.5 . The IP packet size used is 500 bytes, while MAC frames are of 48 bytes with additional 5 bytes due to header (ATM case).

Figure 8.5 shows the queuing delay for each service class, while Figure 8.6 presents the corresponding delay ratios under constant bit-rate traffic [10]. The ideal value for the ratios is 0.5 for all cases. From the plotted results, it is clear that the SWTP scheduler can indeed emulate closely the PDS model. Since the PDS model requires that the 'spacing' between any two service classes strictly adheres to the ratio of the DDPs for the specified service classes, the scheduler should not be dependent on the traffic distribution between service classes. Figure 8.7 [10] shows the result of this test at a utilization of $95 \%$ : the achieved ratios are very near to the ideal value of 0.5 .

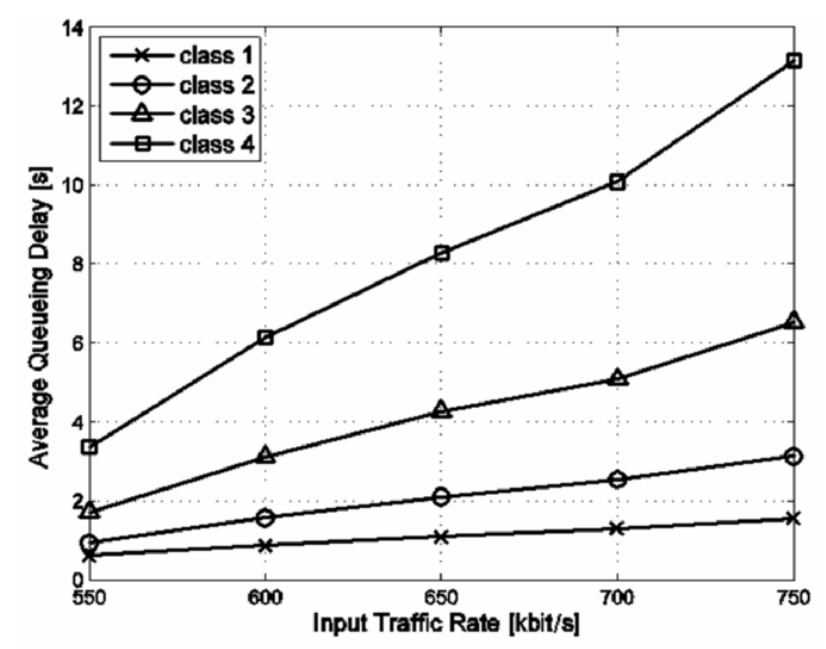

Fig. 8.5: Queuing delay of different service classes following the specified spacing of the model. See reference [10]. Copyright (C)2005 IEEE. 


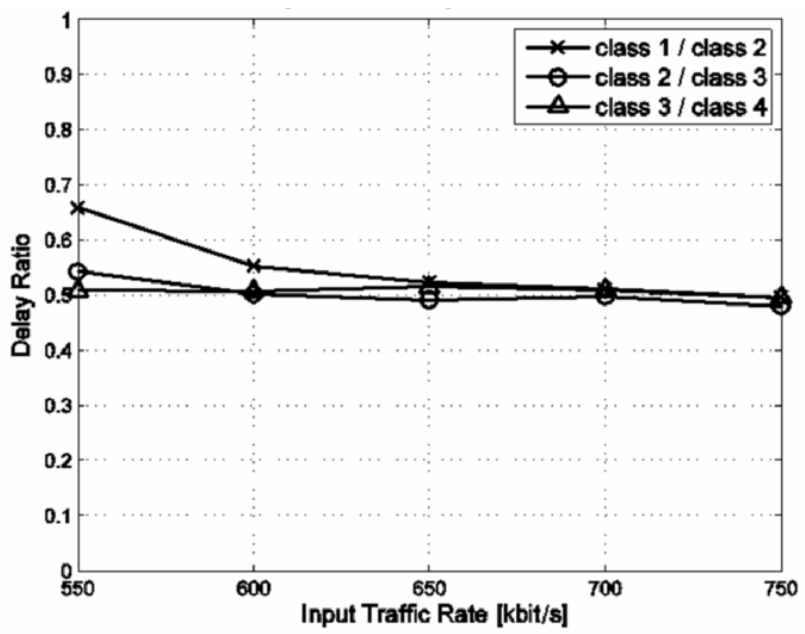

Fig. 8.6: Delay ratios achieved that are close to the ideal delay ratios. See reference [10]. Copyright (C)2005 IEEE.

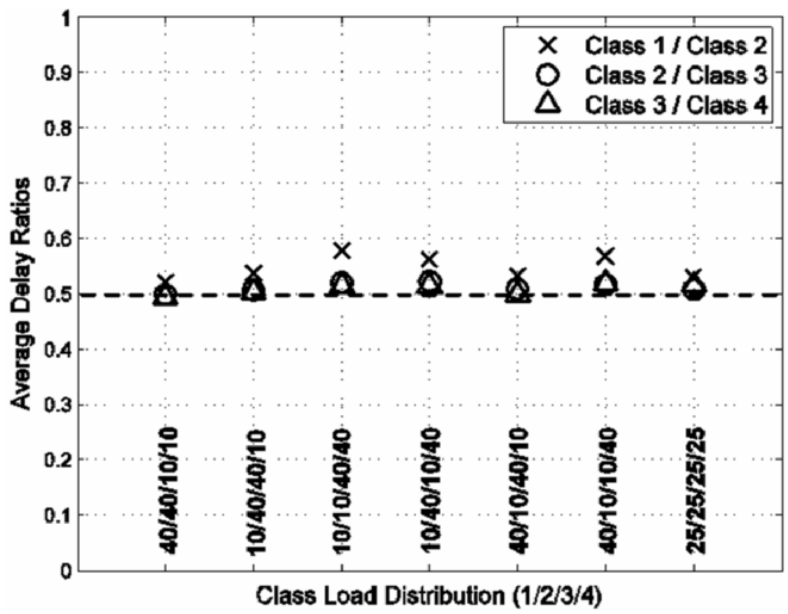

Fig. 8.7: SWTP emulating the PDS in different load distributions with all values achieved close to the ideal value. See reference [10]. Copyright (C)2005 IEEE. 
To illustrate the capability of SWTP in accurately controlling the spacing between different service classes, three sets of DDPs have been defined below.

- Set A: $[1,1 / 2,1 / 4,1 / 8]$

- Set B: $[1,1 / 2,1 / 3,1 / 4]$

- Set C: $[1,1 / 4,1 / 5,1 / 6]$.

Simulations with utilization of $95 \%$ have been conducted based on these DDP sets and the results given in Figure 8.8 [10] show the normalized ratios of all the three cases, where the normalized ratios are defined as the achieved performance ratios divided by the respective ideal ratios. With the ideal value as 1.0, it can be concluded that SWTP is indeed able to control the class spacing. However, due to the long propagation delay, the spacing between the highest and lowest DDP should not be too large to ensure reasonable delay for the lowest class.

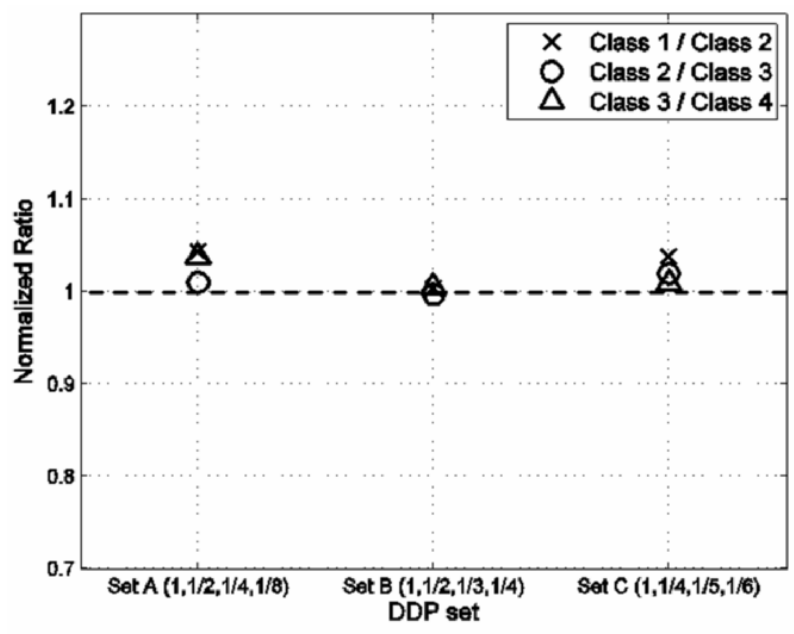

Fig. 8.8: SWTP with 3 sets of DDPs: all normalized delay ratios are close to the ideal value. See reference [10]. Copyright (C)2005 IEEE.

The behavior of SWTP in short timescale is investigated to ensure that the predictability requirement of the PDS model is satisfied. Figure 8.9 [10] shows the individual packet delays upon departure in a four-class scenario for a period of $100 \mathrm{~ms}$. The graph shows that SWTP can consistently provide the appropriate spacing for the service classes. 


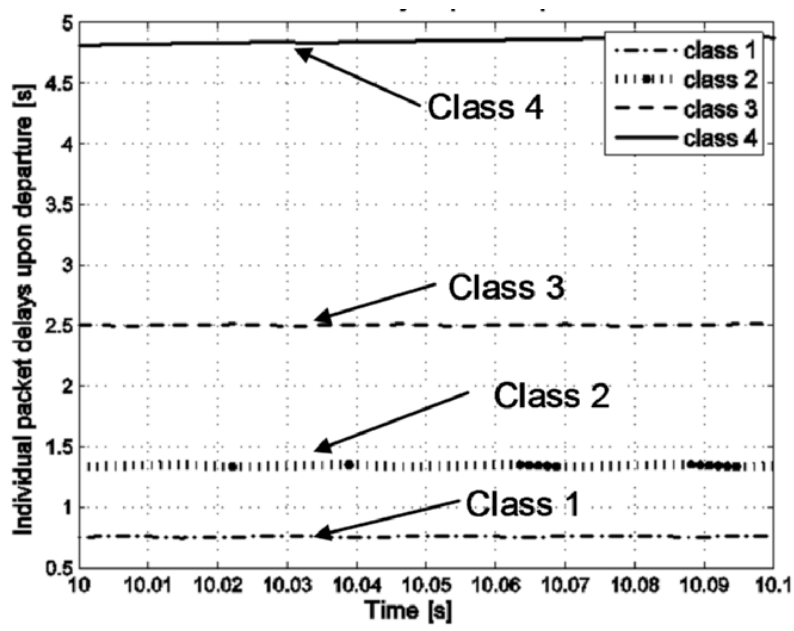

Fig. 8.9: Short time scale behavior of SWTP showing its predictability property. See reference [10]. Copyright (C)2005 IEEE.

\subsection{QoS mapping over satellite-independent service access point}

In what follows, we are specifically concerned with the cross-layer interaction between the network and the MAC layer, in order to preserve QoS requirements, or, in more precise terms, to operate a mapping between the QoS mechanisms operating at the two layers. Within a more general view, with reference to the ETSI Broadband Satellite Multimedia (BSM) protocol architecture [15],[16], we might refer to the inter-working between the Satellite-Independent (SI) and the Satellite-Dependent (SD) architectural components at the SI-SAP (Satellite-Independent - Service Access Point), by taking into account both the change in encapsulation format and the traffic aggregation in the passage from SI to SD queues. Note that the ETSI BSM architecture has been described in Chapter 1, Section 1.5.

Cross-layer RRM problems, involving network and MAC layers, have been extensively considered in [17]-[19]. Reference [20] also provides guidelines and architectural details. In particular, in [17]-[19] Dynamic Bandwidth Allocation (DBA) is applied by computing bandwidth requests for each Earth station's DiffServ queue, which are passed to a centralized scheduler, typically residing in a Master Control Station (MCS). The latter assigns the bandwidth proportionally to the requests received; the remaining capacity is assigned on a free basis. Such scheme has been called Combined Free/Demand Assignment Multiple Access (CF/DAMA).

In a similar context, the problem of QoS mapping between adjacent layers has been recently treated in [21]-[23]. Rather than considering specifically the 
network and the MAC layers, the problem is posed in the more general ETSI BSM scenario mentioned above. In the presence of IP DiffServ queues at the higher layer, the problem consists in dynamically assigning the bandwidth (service rate) to each SD queue, so that the performance required at the IP layer is guaranteed. By considering a fluid model and the loss volume as the performance indicator of interest, the Infinitesimal Perturbation Analysis (IPA) technique of Cassandras et al. [24] (already mentioned in Chapter 7 in a different scenario) is applied in order to maintain on-line the equalization between the loss volumes at the two different layers (by assuming that the resource allocation at the SI layer is capable of satisfying the requirements). In doing so, both traffic and fading variations are taken into account. Further details on the application of the IPA technique are provided in sub-Section 8.4.2.

\subsubsection{Model-based techniques for QoS mapping and support}

Earth stations use reservation mechanisms (bandwidth requests) to transmit their traffic flows (voice or MPEG video, bandwidth reserved for DiffServ aggregates, MPLS pipes, etc.), which may be carried with priority at the satellite link level within some specific DVB service classes. The control process works upon requests for bandwidth allocation, which can be satisfied within a Round Trip Time (RTT) for the request to reach the scheduler and the response to be received (referred to as DBA cycle time in [17]). Hence, whenever traffic flows are characterized by a relatively low burstiness (e.g., the peak-to-average ratio of their rates is close to 1), simple DAMA schemes (e.g., VBDC) can be employed to manage the traffic of Earth stations [19]. The bandwidth allocation can be controlled in this case by means of CAC functions. When burstiness is higher, DBA is applied by computing bandwidth requests (on the basis of a model) for each Earth station's DiffServ queue, which are passed to a centralized scheduler that assigns the bandwidth proportionally to the requests received; the remaining capacity is assigned on a free basis, according to CF/DAMA. Various traffic models have been used to represent the burst-level behavior of real-time Variable Bit Rate (VBR) traffic; among them, we can consider voice with silence detection and VBR-encoded MPEG video. In this case, two control functionalities at different time scales should be employed, namely, CAC at the call level and DBA at the burst level, to guarantee at the same time both a specified degree of QoS and an efficient bandwidth utilization.

In [17], models capturing both Short Range Dependent (SRD) and Long Range Dependent (LRD) behaviors have been used to represent the arrival processes of traffic aggregates to the User Terminal (UT) IP queues in a DiffServ scenario. They are based on Markov-Modulated Poisson Processes (MMPP) and Pareto-Modulated Poisson Processes (PMPP), giving rise to MMPP/G/1 and PMPP/G/1 queuing systems, respectively. The adopted service-dependent QoS metric is the probability that the length of each 
service queue exceeds a given threshold; we consider the constraint that this probability must be kept below a specified value, beyond which the station is considered in outage. The scheduling of the MAC queues must be such that this constraint is fulfilled for the IP-level queues (i.e., those corresponding to $\mathrm{EF}, \mathrm{AF}$ and $\mathrm{BE}$ services within a given Earth station). No fading variations are taken into account, but, as noted in [17], the effect of fade countermeasures might be included as a reduction in the available uplink bandwidth. Note that if the state of the sources can be assumed to change more slowly than the DBA cycle time, within which the allocated bandwidth remains constant, the queuing behavior in these intervals can be approximated by a much simpler $\mathrm{M} / \mathrm{D} / 1$ system.

\subsubsection{A measurement-based approach for QoS mapping and support}

The work done in [21]-[23] takes a different look at the QoS mapping and support problem, by disregarding the use of models, but rather relying on measurement-based optimization techniques. This framework is that of ETSIBSM [15],[16] (let us consider for example the RBDC scheme). In such a context, two basic facts are taken into account: the change of information unit (e.g., from IP to IP-over-DVB) and the heterogeneous traffic aggregation, since, for hardware implementation constraints, the number of available SD queues can be lower than that of SI queues (see also Chapter 1, sub-Section 1.4.3). Figure 8.10, taken from [21], reports and example.

The problem is then how much bandwidth must be assigned to each SD queue, so that the SI IP-based SLA (i.e., the performance expected) is guaranteed. In doing this, the effect of fading on the satellite channel is also taken into account. As in other works (see, e.g., [25]), when the fade countermeasure in use is modulation and coding rate adaptation, the effect of fading is modeled as a reduction in the bandwidth (i.e., the service rate) effectively 'seen' by a layer 2 traffic buffer.

IP Packet Loss Probability (PLP) is one of the SLA performance metrics considered in [23] (the other being IP Packet Average Delay). However, we concentrate here on PLP. The mathematical framework is based on Stochastic Fluid Models (SFM) of the SI-SAP traffic buffers [24],[26]. $N$ SI queues and, without loss of generality, one single SD queue are considered for the analytical formulation (Figure 8.11).

Let $\alpha_{i}^{S I}(t)$ be the input process entering the $i$-th traffic buffer at the SI layer at time $t, i=1, \ldots, N$. After entering one single buffer [with service rate $\left.\theta_{i}^{S I}(t)\right]$ at the SI layer, each $\alpha_{i}^{S I}(t)$ process is conveyed to a single SD buffer [whose service rate is $\theta^{S D}(t)$ ] at the SD layer after a format change. ${ }^{i} L_{V}^{S I}\left[\alpha_{i}^{S I}(t), \theta_{i}^{S I}(t)\right]$ denotes the loss volume of the $i$-th IP buffer according to the bandwidth allocation $\theta_{i}^{S I}(t)$.

Let $\alpha^{S D}(t)$ be the input process of the buffer at the SD layer at time $t$. The $\alpha^{S D}(t)$ process derives from the output processes of the SI buffers. 


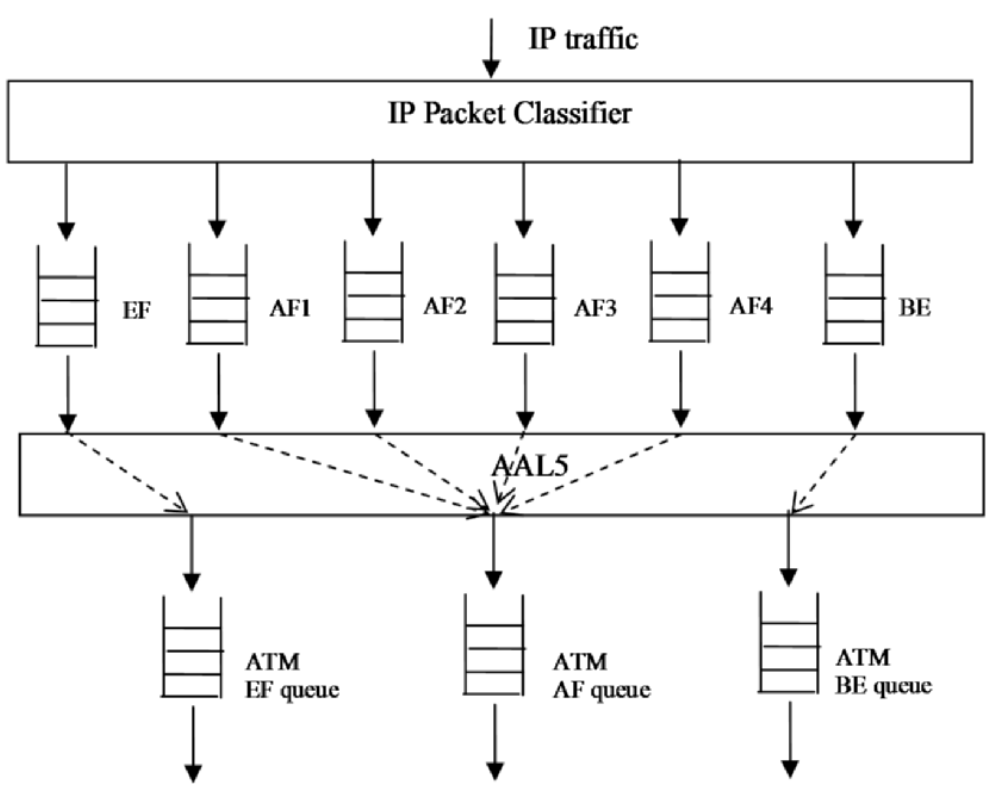

Fig. 8.10: Queuing at the SI-SAP interface: satellite-independent (DiffServ) over satellite-dependent layer (ATM). See reference [21]. Copyright (C2005 IEEE.

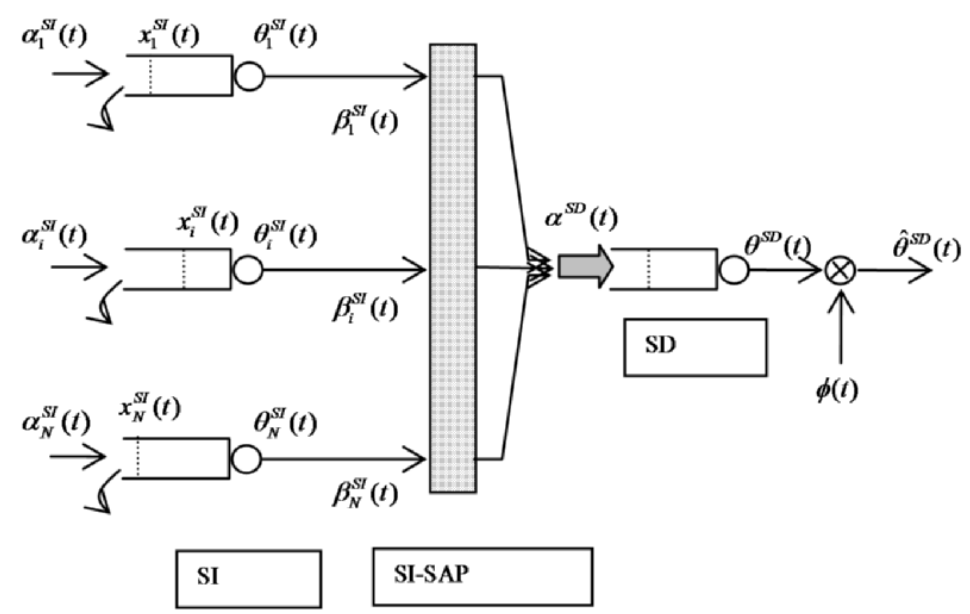

Fig. 8.11: Stochastic processes and buffer set for the envisaged SI-SAP queuing model. 
The loss volume of the $i$-th traffic class within the SD buffer is indicated by ${ }^{i} L_{V}^{S D}\left[\alpha^{S D}(t), \theta^{S D}(t) \cdot \phi(t)\right]$. It is a function of the following elements: the SD input process $\alpha^{S D}(t)$, the fading process $\phi(t)$ and the SD bandwidth allocation $\theta^{S D}(t)$. It is remarkable that ${ }^{i} L_{V}^{S D}(\cdot)$ cannot be obtained in closed-from.

The problem reveals to be the equalization of the QoS measured at the different layers of the protocol stack (i.e., SI and SD):

QoS Mapping Optimization (QoSMO) Problem: find the optimal bandwidth allocation, ${ }^{O p t} \theta^{S D}(t)$, so that the cost function $J\left(\cdot, \theta^{S D}(t)\right)$ is minimized:

$$
\begin{gathered}
{ }^{O p t} \theta^{S D}(t)=\arg \min _{\theta^{\mathrm{SD}}(t)} J\left(\cdot, \theta^{S D}(t)\right) ; J\left(\cdot, \theta^{S D}(t)\right)=\underset{\omega \in \Theta}{E} L_{\Delta V}\left(\cdot, \theta^{S D}(t)\right) \\
L_{\Delta V}\left(\cdot, \theta^{S D}(t)\right)=\sum_{i=1}^{N}\left[{ }^{i} L_{V}^{S I}\left(\alpha_{i}^{S I}(t), \theta_{i}^{S I}(t)\right)-{ }^{i} L_{V}^{S D}\left(\alpha^{S D}(t), \theta^{S D}(t) \cdot \phi(t)\right)\right]^{2} .
\end{gathered}
$$

In (8.3), $\omega$ denotes a sample path of the system, i.e., a realization of the stochastic processes involved in the problem (coming from quantities $\phi(t)$, $\left.\alpha_{i}^{S I}(t), i=1, \ldots, N, \alpha^{S D}(t)\right)$. Note that the cost function [see the second row in (8.3)] weighs the sum of the quadratic deviations of the loss volumes at the two layers, over all traffic classes associated with SI queues.

This QoSMO problem is very complex to be solved. Two approaches are considered below; one is based on the equivalent bandwidth concept and the other is based on IPA.

Traditionally, equivalent bandwidth techniques are based on the statistical characterization of the traffic generated by users' applications. The only simply applicable statistics, useful for the SD rate provision, are the mean $(m)$ and the standard deviation $(\sigma)$ of the $\alpha^{S D}$ process. Hence, a popular equivalent bandwidth technique, actually applicable in this context, is ruled by (8.4) below [27]. Let us consider the following notations: $k=1,2, \ldots$ the time instants of the SD rate reallocations, $m_{\alpha^{S D}}(k)$ and $\sigma_{\alpha S D}(k)$ the mean and the standard deviation, respectively, of the SD input process measured over the time interval $[k, k+1]$. Therefore, the bandwidth provision $\theta^{S D}(k+1)$ at the SD layer, assigned for the time interval $[k+1, k+2]$, may be computed as:

$$
\theta^{S D}(k+1)=m_{\alpha^{S D}}(k)+a \cdot \sigma_{\alpha^{S D}}(k)
$$

where $a=\sqrt{-2 \ln (\varepsilon)-\ln (2 \pi)}$ and $\varepsilon$ represents the upper bound on the allowed PLP. Such allocation method is called Equivalent Bandwidth approach $(\mathrm{EqB})$ in what follows.

In [23], another measurement-based equivalent bandwidth algorithm is proposed that can face: 
- Heterogeneity of the QoS requests in the aggregated trunk;

- Change of encapsulation format;

- Fading counteraction;

- No knowledge of SD input process's statistical properties;

- No knowledge of SD buffer size.

To match these requirements, the derivative of the cost function $L_{\Delta V}(\cdot)$ is used:

$$
\frac{\partial L_{\Delta V}\left(\cdot, \theta^{S D}\right)}{\partial \theta^{S D}}=2 \cdot \sum_{i=1}^{N} \frac{\partial^{i} L_{V}^{S D}\left(\theta^{S D}\right)}{\partial \theta^{S D}}\left[{ }^{i} L_{V}^{S D}\left(\theta^{S D}\right)-{ }^{i} L_{V}^{S I}\left(\theta_{i}^{S I}\right)\right] .
$$

Using IPA (see, e.g., [24],[26] and references therein), each $\frac{\partial^{i} L_{V}^{S D}\left(\theta^{S D}\right)}{\partial \theta^{S D}}$ component can be obtained in real-time only on the basis of some traffic samples acquired during the system evolution. Let $[k, k+1]$ be the time interval between two consecutive SD bandwidth reallocations. The interval of time in which the buffer is not empty are defined as busy periods. The derivative estimation is computed at the end of the decision epoch $[k, k+1]$ as follows:

$$
\begin{aligned}
& \left.\frac{\partial^{i} L_{V}^{S D}\left(\theta^{S D}\right)}{\partial \theta^{S D}}\right|_{\hat{\theta} S D(k)}=\left.\phi(k) \cdot \sum_{\varsigma=1}^{N_{k}^{i}} \frac{\partial^{i} L_{k, \varsigma}^{S D}\left(\theta^{S D}\right)}{\partial \theta^{S D}}\right|_{\hat{\theta}}{ }^{S D(k)} \\
& \left.\frac{\partial^{i} L_{k, \varsigma}^{S D}\left(\theta^{S D}\right)}{\partial \theta^{S D}}\right|_{\hat{\theta}^{S D}(k)}=-\left({ }^{i} \nu_{\varsigma}^{k}\left(\hat{\theta}^{S D}(k)\right)-{ }^{i} \xi_{\varsigma}^{k}\left(\hat{\theta}^{S D}(k)\right)\right)
\end{aligned}
$$

where ${ }^{i} L_{k, \varsigma}^{S D}\left(\theta^{S D}\right)$ is the $\varsigma$-th contribution to the SD loss volume of the $i$-th traffic class for each busy period $B_{k}^{\varsigma}$ within the decision interval $[k, k+1]$; $\xi_{\varsigma}^{k}$ is the starting point of $B_{k}^{\varsigma} ; \nu_{\varsigma}^{k}$ is the instant of time when the last loss occurs during $B_{k}^{\varsigma} ; N_{k}^{i}$ is the number of busy periods within the interval $[k$, $k+1]$ for service class $i$. It must be noted that $\hat{\theta}^{S D}(k)$ represents the SD bandwidth reduction due to fading within the time interval $[k, k+1]$ (i.e., $\hat{\theta}^{S D}(k)=\theta^{S D}(k) \cdot \phi(k)$, where $\phi(k)$ represents the bandwidth reduction seen at the SD layer, due to redundancy applied at the physical layer to counteract channel degradation).

The proposed optimization algorithm is based on the gradient method, whose descent step is ruled by (8.8):

$$
\theta^{S D}(k+1)=\theta^{S D}(k)-\left.\eta_{k} \cdot \frac{\partial L_{\Delta V}\left(\cdot, \theta^{S D}\right)}{\partial \theta^{S D}}\right|_{\hat{\theta}^{S D}(k)} \quad ; \quad k=1,2, \ldots
$$

In (8.8), $\eta_{k}$ denotes the gradient step size and $k$ the reallocation time instant. This method is called Reference Chaser Bandwidth Controller (RCBC). 


\subsubsection{Performance evaluation and discussion}

These rate control mechanisms (i.e., $\mathrm{RCBC}$ and $\mathrm{EqB}$ ) have been investigated through simulations [21],[23]. An ad-hoc $\mathrm{C}++$ simulator has been developed for the SI-SAP environment described above, considering a general satellite system. In what follows, for the sake of simplicity, only the traffic aggregation problem is faced by assuming no channel degradation over the satellite channel.

The case considered is that of two SI traffic buffers. The first one conveys the traffic of 30 VoIP sources. Each VoIP source is modeled as an exponentially-modulated on-off process, with mean "on" and "off" times equal to $1.008 \mathrm{~s}$ and $1.587 \mathrm{~s}$, respectively. All VoIP connections have peak rate of $64 \mathrm{kbit} / \mathrm{s}$. The IP packet size is 80 bytes. The SI service rate for VoIP assures an SLA target PLP below $10^{-2}$ (SI VoIP buffer size is 30 IP packets). The second buffer is dedicated to a video service. "Jurassic Park I" video trace, taken from the Web site referenced in [28], is used. The SI rate allocation for video (also measured through simulations), is $350 \mathrm{kbit} / \mathrm{s}$. It assures a PLP $=10^{-3}$, which is the target SLA for video (the SI video buffer size is 10,500 bytes). Both outputs of the SI buffers are conveyed towards a single queue at the SD layer. DVB encapsulation (header 4 bytes, payload 184 bytes) of the IP packets through the LLC/SNAP (overhead 8 bytes) is implemented in this case. The SD buffer size is 300 DVB cells.

In Figure 8.12 (firstly presented in [21]), the SD bandwidth provision produced by $\mathrm{RCBC}$ is compared with $\mathrm{EqB}$. The loss probability bound $\varepsilon$ for $\mathrm{EqB}$ is set to $10^{-3}$, being the most stringent PLP constraint imposed at the SI level. The time interval between two consecutive SD bandwidth reallocations is denoted by $T_{R C B C}$ and $T_{E q B}$, for RCBC and EqB respectively. Note that in the following graphs, for the sake of simplicity, $T$ denotes $T_{R C B C}\left(T_{E q B}\right)$ in the $\mathrm{RCBC}(\mathrm{EqB})$ case.

$T_{R C B C}$ is fixed to 7 minutes, while $T_{E q B}$ is set to the following values:

$$
\left\{T_{R C B C} \cdot 1 / 3, T_{R C B C} \cdot 1 / 2, T_{R C B C}, T_{R C B C} \cdot 2, T_{R C B C} \cdot 4\right\}
$$

in different tests in order to highlight the possible inaccuracy introduced by the real-time computation of the EqB statistics using different time scales.

According to Figure 8.12, RCBC captures the bandwidth needs of the SD layer in a single reallocation step. Whereas, EqB produces strong oscillations in the SD rate assignment. It is also clear from Figure 8.12 that the IPA-based estimation (8.5) is more robust than the on-line estimation of $m_{\alpha^{S D}}$ and $\sigma_{\alpha^{S D}}$. The IPA sensitivity estimation drives RCBC toward the optimal solution of the QoSMO problem.

The SD buffer's video PLP, averaged over the entire simulation horizon, is shown in Figure 8.13 (taken from [21]). The performance of RCBC, referenced to as "SD layer RCBC" is very satisfying: actually, the RCBC video PLP is $7.56 \cdot 10^{-4}$. A result "below threshold" has been measured for 


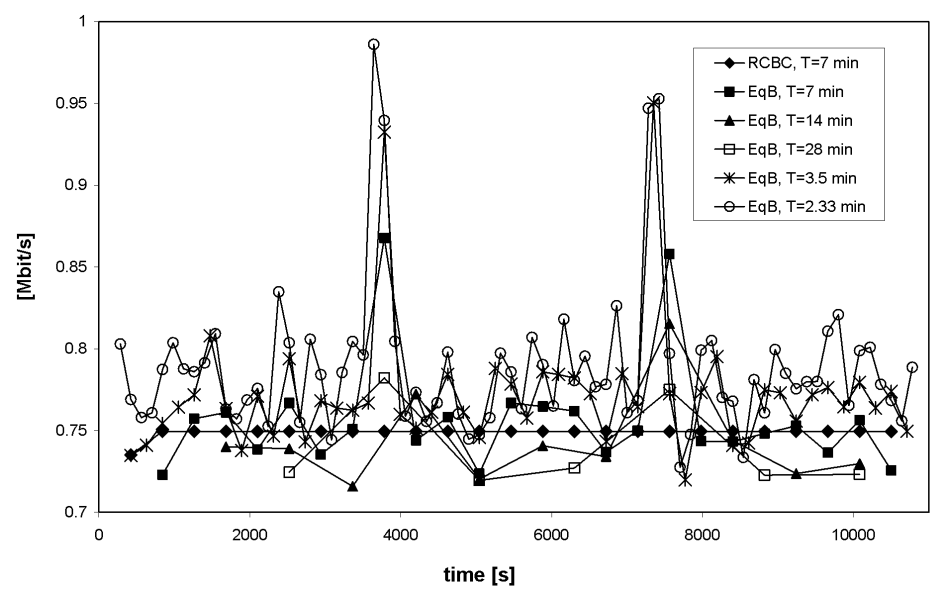

Fig. 8.12: Aggregation of VoIP and Video. SD allocations. RCBC versus EqB. See reference [21]. Copyright (C)2005 IEEE.

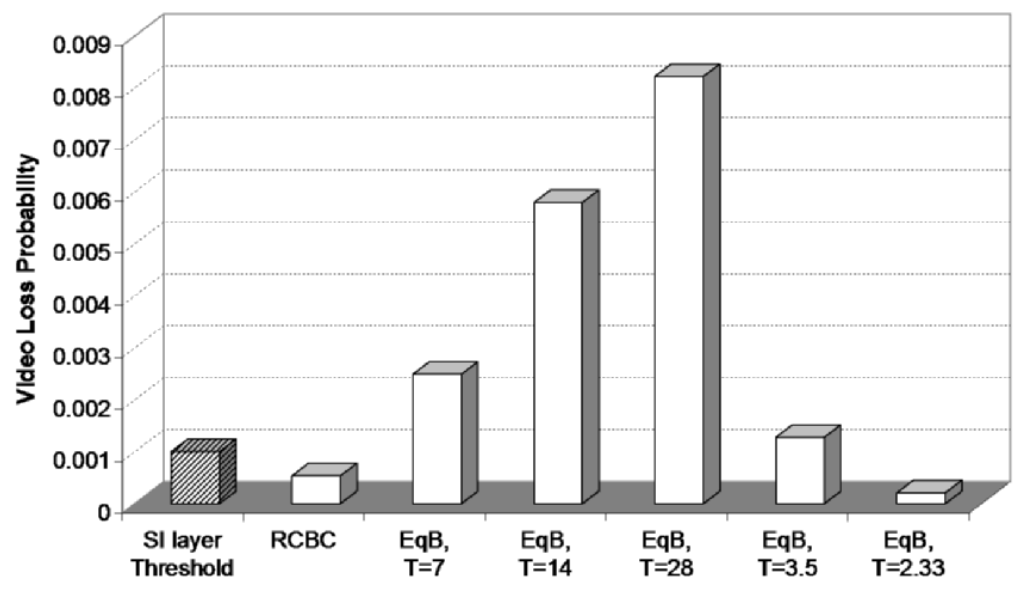

Fig. 8.13: Aggregation of VoIP and Video. Video PLP. See reference [21]. Copyright (C) 2005 IEEE.

EqB only for frequent reallocations $\left(T_{\mathrm{EqB}}=T_{R C B C} \cdot 1 / 3=2.33\right.$ minutes $)$. The corresponding bandwidth allocations, averaged over the simulation duration, are shown in Figure 8.14 (taken from [21]). RCBC not only allows saving bandwidth compared to the "SD layer EqB T $=2.33$ min" strategy, but offers a performance comparable to the other EqB cases, whose offered PLP is far from the SI threshold. In brief, RCBC finds the optimal operation point of the system, namely, the minimum SD bandwidth provision needed to track the SI QoS thresholds. 


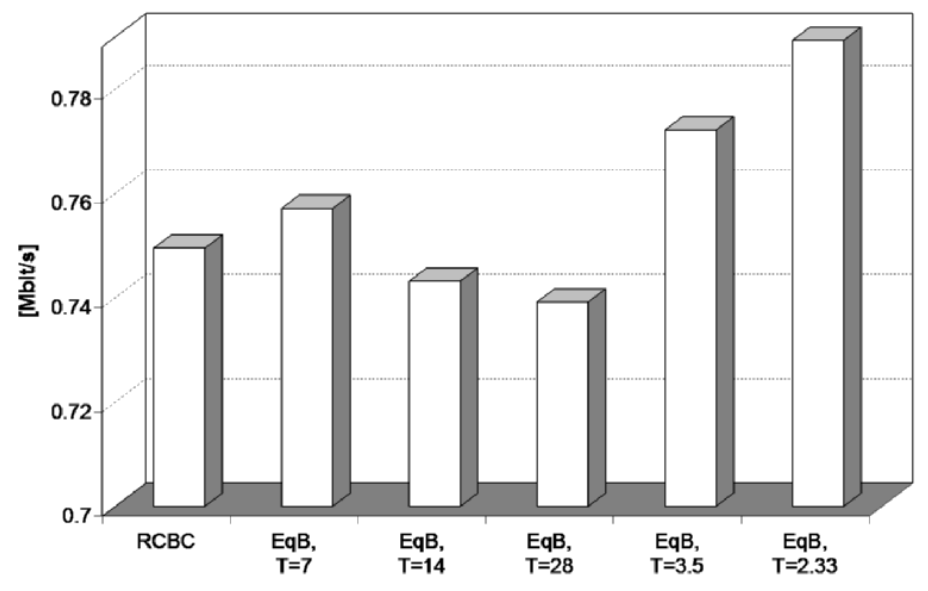

Fig. 8.14: Aggregation of VoIP and Video. Average SD bandwidth provision. See reference [21]. Copyright (C)2005 IEEE.

\subsection{QoS provisioning for terminals supporting dual network access - satellite and terrestrial}

When terminals support dual network access -satellite and terrestrial (WLAN, UMTS, etc.) links- it is quite critical to select the appropriate network for each application, depending on both the resources available and the kind of application involved. In some instances (such as real-time tele-operation), it is not only a matter of user satisfaction, but also of satisfying critical service goals. For example, the QoS provision may be related to the deadline fulfillment: violating a deadline may cause a sea farm hitting the sea bottom or a remote probe bump into a rock.

This Section provides an analysis on relevant technologies in this context and focuses on QoS frameworks to support terminal mobility between satellite, wireless, and terrestrial networks. In particular, we analyze the problem of the multiple access to different networks (which includes satellite, wireless, and terrestrial networks) in order to support more than one access network at the same time. In such a context, the focus is on network selection based on QoS parameters. We work on QoS parameter identification at layer 2 for selected applications as well as IP-oriented solutions for network mobility and network selection. Let us consider two specific topics:

- Redundant codes in hybrid networks and

- Mechanisms for error recovery in WiFi access points. 


\section{Redundant codes in hybrid networks}

Hybrid networks consisting of satellite links and mobile ad hoc networks present a series of challenges due to different packet-loss patterns, delay, and, usually, scarce available bandwidth. In this scenario, redundant encoding, in the form of Forward Erasure Correction (FZC) codes [29],[30], can provide an effective protection against losses in multicast videoconferencing and video streaming applications. The use of efficient encoding techniques can provide further reduction on bandwidth requirements.

A real test-bed based on a remote video streaming server interconnected via a GEO-satellite pipe to a local WLAN (both $11 \mathrm{Mbit} / \mathrm{s}$ and $5 \mathrm{Mbit} / \mathrm{s}$ cases have been considered, according to IEEE 802.11b) is presented in [31], by adopting the multicast network protocol. The satellite pipe is based on the commercial Skyplex network [32] that operates in the Ka band with the Hotbird 6 transponder. The developed platform, described in [33], is shown in Figure 8.15. The purpose is to provide users with a low-cost, high-availability platform for performing experiments with IP packets over the Skyplex platform. Such devices have been also used to experiment the FZC encoding.

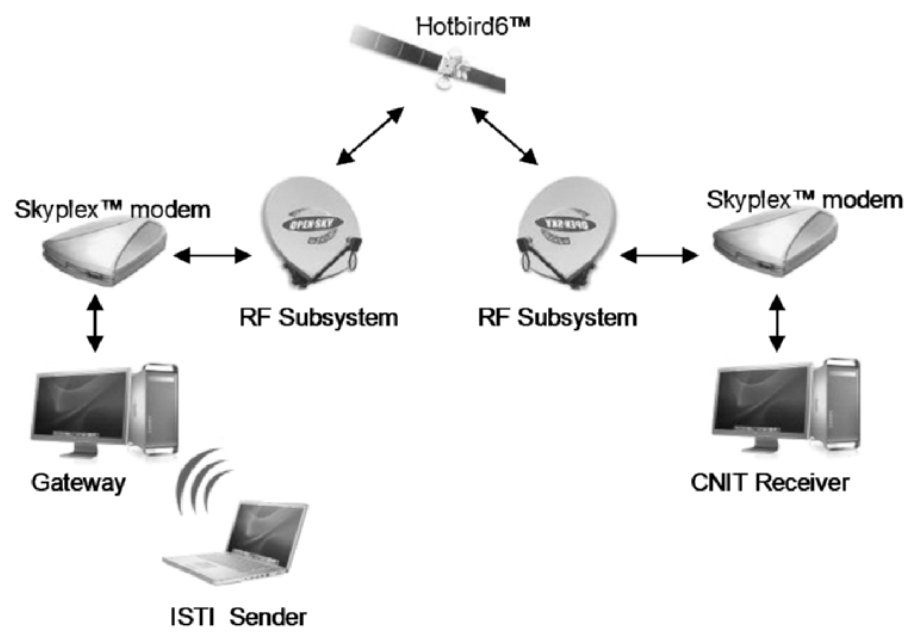

Fig. 8.15: Test-bed platform architecture.

The obtained experimental measurements show the performance of FZC codes based on Vandermonde matrix [34], for multicast video streaming applications. Basically, $k$ blocks of source data are encoded to produce $n$ blocks of encoded data (with $n>k$ ), such that any subset of $k$-encoded blocks suffices to reconstruct the $k$-block source data. Considering the real 
implementation, the encoder works at the transport layer by fetching $k$ information packets from the video stream and then transmitting $k+l$ UDP packets ( $k$ of information $+l$ of redundancy) towards the receiving host. The encoder adds a preamble of 4 bytes for the sequence number, which is then cancelled by the decoder. The decoder fetches $k$ of the $k+l$ packets per block and recovers the information, provided that no more than $l$ packets are lost in a single block of packets. The receiver then feeds the MPEG-4 decoder with the received stream. Different MPEG-4 movies have been broadcast towards the terrestrial WLAN through the satellite channel by using a standard video decoder and home-made FZC encoding/decoding software. The authors in [31] used this software to work between the application layer and the UDP transport layer; however, this software can also be used between layer 2 and 3 .

The performance evaluation has been based both on subjective perception of QoS and on objective parameters of QoS. The used subjective assessment has been the perceptual quality, called the Mean Opinion Score (MOS), which requires several observers and many tests in order to provide a reasonable statistical spread of results $\left({ }^{1}\right)$. The reference measure used for an objective video quality assessment has been the Peak Signal to Noise Ratio (PSNR), calculated on the difference signal between the original error-free sequence and the received decoded sequence. Other general objective parameters of QoS, such as packet delivery delay and packet loss, have been considered in [31]. In what follows the numerical results are presented.

Packet Loss - The experiment in un-coded mode (no FZC) shows that packet loss can be reduced from $13 \%$ to about $6 \%$ by changing the transmission rate from 11 to $5.5 \mathrm{Mbit} / \mathrm{s}$ (see Table 8.1). In this case, the channel occupancy increases by $56 \%$. Interestingly, we can note that with FZC and 200/100 coding ratio the packet loss is almost zero.

\begin{tabular}{|c|c|c|}
\hline Coding ratio & Packet Loss & Residual Bandwidth [Mbit/s] \\
\hline Uncoded 11 Mbit/s & $12.98 \%$ & 4.77 \\
\hline $110 / 100$ & $8.17 \%$ & 4.67 \\
\hline Uncoded 5.5 Mbit/s & $5.39 \%$ & 4.24 \\
\hline $120 / 100$ & $3.25 \%$ & 4.58 \\
\hline $130 / 100$ & $0.83 \%$ & 4.48 \\
\hline $200 / 100$ & $0 \%$ & 3.83 \\
\hline
\end{tabular}

Table 8.1: Packet loss and residual bandwidth after FZC encoding.

\footnotetext{
${ }^{1}$ According to ITU-R Recommendation 500-5, MOS values are: imperceptible (5), perceptible but not annoying (4), slightly annoying (3), annoying (2), very annoying (1).
} 
Packet delivery delay - The maximum packet delivery delay is evaluated as the time necessary to recover all the information when a number of packets equal to the redundancy packets are lost. Table 8.2 shows the packet delivery delay versus the coding ratio.

\begin{tabular}{|c|c|c|c|}
\hline Coding ratio & Max [ms] & Mean [ms] & Var. $\left[\mathbf{m s}^{2}\right]$ \\
\hline $110 / 100$ & 105.6 & 52.149 & 0.769 \\
\hline $120 / 100$ & 115.2 & 54.99 & 0.792 \\
\hline $130 / 100$ & 124.8 & 52.637 & 0.805 \\
\hline $200 / 100$ & 192 & 53.805 & 1.675 \\
\hline
\end{tabular}

Table 8.2: Maximum, mean and variance of delivery delay. See reference [31]. Copyright (C)2005 IEEE.

Mean Opinion Score (MOS) - Thirty persons have answered to three quality questions (overall, video, and audio quality) for each considered coding ratio; MOSs have been calculated. The percentages of people, who have considered the video acceptable, are shown in Table 8.3.

\begin{tabular}{|c|c|c|c|c|c|}
\hline Uncoded & $\mathbf{1 1 0 / 1 0 0}$ & Uncoded & $\mathbf{1 2 0 / 1 0 0}$ & $\mathbf{1 3 0 / 1 0 0}$ & $\mathbf{2 0 0 / 1 0 0}$ \\
$\mathbf{1 1} \mathbf{M b i t} / \mathbf{s}$ & $\mathbf{1 1} \mathbf{M b i t} / \mathbf{s}$ & $\mathbf{5 . 5} \mathbf{M b i t} / \mathbf{s}$ & $\mathbf{1 1} \mathbf{M b i t} / \mathbf{s}$ & $\mathbf{1 1} \mathbf{M b i t} / \mathbf{s}$ & $\mathbf{1 1} \mathbf{M b i t} / \mathbf{s}$ \\
\hline $7.7 \%$ & $15.4 \%$ & $26.9 \%$ & $34.6 \%$ & $100 \%$ & $100 \%$ \\
\hline
\end{tabular}

Table 8.3: Acceptability of received video. See reference [31]. Copyright (C)2005 IEEE.

PSNR - The PSNR-based video quality metric (henceforth denoted as $\mathrm{VQM}_{P}$ - Peak Video Quality Measurement) uses a form of the logistics function that is recommended in references [35],[36] and evaluates the mean of how much transmitted frames differ from the original ones. Sixty seconds of the transmitted video have been compared with the received video to evaluate the $\mathrm{VQM}_{P}$ parameter. Results are presented in Figure 8.16, where $\mathrm{VQM}_{P}$ is compared for different coding ratio values.

\section{Error recovery in $\mathrm{WiFi}$ access points}

The second topic of our study on the interconnection of WLAN and satellite networks deal with some mechanisms for error recovery when a Fast HandOver (FHO) occurs between different IEEE 802.11b Access Points (APs). Fast handover techniques using paradigms like make-before-break or bi-casting reduce the L3 handover time to extremely short delays that are acceptable for all the applications [37]. However, in layer 2 (L2), the handover time is 


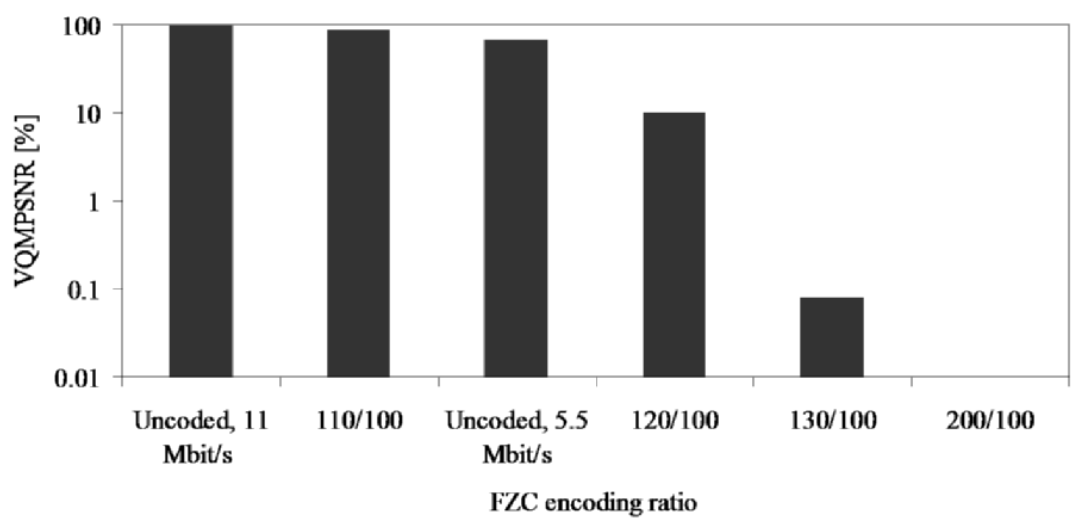

Fig. 8.16: Peak Video Quality Measurement.

very high for some technologies. So, when doing an intra-technology handover (e.g., the interface changes its AP and/or channel), an unacceptable disruption may occur. For instance, in IEEE $802.11 \mathrm{~b}(\mathrm{WiFi})$ an FHO procedure can take from about $500 \mathrm{~ms}$ in a standard mode, to less than $10 \mathrm{~ms}$ in an optimized mode, where the number of frequencies, scanned in order to establish the new communication, is sensibly reduced [38]. During such a time, all transmitted information can be lost. In such a context, the adoption of robust FZC codes can permit to recover totally the lost information. This procedure may cost in terms of bandwidth utilization and computational complexity, due to the generation of the redundant information. To minimize these facts we propose to use FZC in the last hop, i.e., between the Mobile Node (MN) and its Access Router (AR). In what follows, AP and AR terms are used interchangeably. The Core Network (CN) will then not need extra computational power and use more bandwidth in its access link.

During the L2 disruption, both the ARs and the MN can stop sending packets and buffer them and send them when the connectivity is re-established. Indeed, during an $\mathrm{FHO}$, the MNs have means to predict that there is going to be an L2 disruption. For instance, they may know the instant in which the FHO takes place or finishes or its duration, but, perhaps, not with accuracy or some parameters may be unknown. Buffering by its own is not a perfect solution; hence, we propose to complement it with FEC techniques of the FZC type. Our solution consists in adding (or increasing) the FEC used between MN and ARs during the predicted FHO duration. Table 8.4 permits to understand the advantage of FEC techniques with respect to pure buffering. This table depicts an FHO (beginning and end instants of the L2 disruption are indicated) and also shows when the disruption actually happens. If we use buffering, the communication is cut between the disruption indications and then the buffered packets are sent. But using FEC, MN and 
ARs continue sending packets and the communication is cut only during the actual disruption. When this disruption ends, lost packets may be recovered by exploiting FEC capabilities. When "L2 disruption end" is indicated, FEC can be stopped. Of course the advantage of FEC versus buffering depends on how big is the shift between disruption indication and actual disruption.

\begin{tabular}{|c|c|c|c|c|c|c|}
\hline $\begin{array}{c}\text { TIME } \rightarrow \\
\text { status (mobile } \\
\text { node) }\end{array}$ & $\begin{array}{c}\text { Normal } \\
\text { Tx }\end{array}$ & $\begin{array}{c}\text { L2 } \\
\text { disruption } \\
\text { indication }\end{array}$ & $\begin{array}{c}\text { L2 } \\
\text { disruption }\end{array}$ & $\begin{array}{c}\text { L2 } \\
\text { disruption }\end{array}$ & $\begin{array}{c}\text { L2 } \\
\text { disruption } \\
\text { end } \\
\text { indication }\end{array}$ & $\begin{array}{c}\text { Normal } \\
\text { Tx }\end{array}$ \\
\hline $\begin{array}{c}\text { Mobile node } \\
\text { behavior }\end{array}$ & Buffering & Buffering & Buffering & Buffering & \\
\hline $\begin{array}{c}\text { Correspondent } \\
\text { node behavior }\end{array}$ & pkt Rx & & & & pkt Rx \\
\hline $\begin{array}{c}\text { Mobile node } \\
\text { behavior using } \\
\text { FEC techniques }\end{array}$ & FEC & FEC & FEC & FEC & \\
\hline $\begin{array}{c}\text { Correspondent } \\
\text { node behavior } \\
\text { when the MN } \\
\text { uses FEC during } \\
\text { handover }\end{array}$ & pkt Rx & pkt Rx & & & & pkt Rx \\
\hline
\end{tabular}

Table 8.4: Buffering versus FEC techniques while the transmitter node undergoes an $\mathrm{FHO}$

A first problem to solve is how the MN will indicate its own software and the AR that it is going to perform an FHO. That may depend on the actual L2 technology and even, in some technologies, the AR will be the one telling the $\mathrm{MN}$ that it has to move. In $\mathrm{WiFi}$, when the MN detects that the signal level of its current AR decreases bellow a threshold, it initiates the FHO procedure (scanning new channels in new ARs and then doing the FHO to the selected channels). This issue can trigger two actions in the MN: it starts doing FEC and tells the AR to do so as well.

The second issue to solve is to determine the ideal amount of redundancy in the FEC technique. Hence, we must calculate the maximum number of packets lost during L2 disruption (we must estimate the total disruption time and the packet rate). This number of packets is the redundancy that must be included in the FEC. In Table 8.5 this disruption time (shadowed parts) corresponds to 3 packets and thus 3-packet redundancy is added (packets 3,4 and 5). Note that information packets are labeled with a letter and redundancy packets with a number.

Also the buffering needed at the receiver (both MN side and the AR one, 
depending on the direction of transmission) must be determined. For doing so, we suppose the worst case scenario, when the disruption occurs while sending the information packets and not when sending redundancy packets. In such a case, to recover the information, the whole block composed of information and redundancy packets (for instance block formed by packets D to 5 ), must be received before being able to extract the information packets. This determines the minimum buffering time of 8 packets in Table 8.5.

\begin{tabular}{|c|c|c|c|c|c|c|c|c|c|c|c|c|c|c|c|c|c|c|c|c|c|c|c|}
\hline Time & 5 & \begin{tabular}{|l|l|}
6 & 7
\end{tabular} & & 9 & 0 & 1 & 2 & 34 & 5 & \begin{tabular}{|l|l}
6 & 7
\end{tabular} & 8 & 9 & 0 & 1 & 2 & 3 & \begin{tabular}{|l|l|}
4 & 5
\end{tabular} & 6 & 7 & & \begin{tabular}{|l|l|}
0 & 1
\end{tabular} & $|2|$ & 3 \\
\hline $\begin{array}{l}\text { Informat. } \\
\text { frames }\end{array}$ & $\mathrm{A}$ & B & & $\mathrm{C}$ & & & $\mathrm{D}$ & & $\mathrm{E}$ & $\mathrm{F}$ & & & G & & & $\mathrm{H}$ & & I & & $\mathrm{J}$ & & & \\
\hline Tx frames & & & & & $\mathrm{A}$ & $\mathrm{B}$ & $\mathrm{C}$ & 12 & & & & $\mathrm{D}$ & $\mathrm{E}$ & $I^{2}$ & $\mathrm{G}$ & 3 & \begin{tabular}{|l|l|}
4 & 5 \\
\end{tabular} & & & \begin{tabular}{l|l}
$\mathrm{H}$ & $\mathrm{I}$ \\
\end{tabular} & $\mathrm{J} 6$ & \begin{tabular}{|l|l|}
5 & 7 \\
\end{tabular} & \\
\hline Rx frames & & & & & $\mathrm{A}$ & B & $\mathrm{C}$ & 12 & & & & & & $\mathrm{~F}$ & $\mathrm{G}$ & 3 & $4 \mid 5$ & & & $\begin{array}{ll}\mathrm{H} \mathrm{I} \\
\end{array}$ & $\mathrm{J} 6$ & \begin{tabular}{|l|l|}
6 & 7 \\
\end{tabular} & \\
\hline Buffer at Rx & & & & & & & & & & & $\overline{\mathrm{A}}$ & & & $\mathrm{B}$ & & & $\mathrm{C}$ & & $\mathrm{D}$ & & $\mathrm{E}$ & & $\mathrm{F}$ \\
\hline
\end{tabular}

Table 8.5: FEC technique example. Shadowed cells correspond to disruption.

Note that these aspects can also apply if the FEC technique is employed between the $\mathrm{CN}$ and the $\mathrm{MN}$, being the ARs transparent to that. However, there are many advantages of employing the FEC technique in the MN-AR link and at link level. First, in our proposed FHO scheme, the AR already must have special functionality like bicasting, thus adding this FEC functionality will not complicate too much the AR. Second, redundancy is only present in the last hop, besides in this last hop, FEC techniques may already be employed because the link (e.g., air link) may be prone to errors, so, perhaps our solution can be implemented just modifying the parameters of the existing FEC. Finally, in a multicast scenario, the source will send the data and the ARs would be the ones to add the appropriate FEC redundancy.

\subsection{Switched Ethernet over LEO satellite: implicit cross-layer design exploiting VLANs}

The aim of this Section is to introduce the possibility of exploiting Switched Ethernet facilities in a LEO network using Inter-Satellite Links (ISLs). We refer here to Scenario 3 (see Chapter 1, sub-Section 1.4.5) for the study carried out in this part of Chapter 8. The interest is to support QoS-aware resource management procedures by tacking into consideration the mapping between logical and physical network topologies (that are both well known), like in the case of terrestrial switched-networks. In fact, the optimization of connection paths can be performed, in a large network scenario, by means of routing algorithms or switching mechanisms. The former solution is particularly suited in case of networks whose topology is not completely known or predictable, 
while the switching approach has been adopted in the scope of single domain networks with known topology, or single-provider-managed area networks (LAN, WAN, MAN). LEO satellite can be classified as an extension of LAN/MAN, in which the network topology changes over time in a regular and predictable way, thus the switching approach can be a natural candidate for network management issues. Furthermore, an Ethernet-like switching solution (i.e., the one proposed by IEEE 802.1) addresses the problem of the interoperation between network layer and the satellite-specific MAC. The mechanism is known as Logical Link Control (LLC), and provides a useful framework to deploy an IP-MAC mapping with QoS control.

IEEE 802.1 standards offer a set of facilities meant to build, operate and manage a network comprising one or more transmission technologies and one or more communication methods (i.e., physical and MAC layers). Bridging of different technologies is obtained by using special devices (i.e., bridges) that are able to translate, when needed, frames from a given MAC format to a different one. In particular, IEEE 802 standards propose a special LLC layer [39], located just above the MAC layer [40], that is common to all network segments and whose frames can be transported in any kind of MAC frames. LLC is the glue that allows the system to interconnect different physical and MAC solutions of the IEEE 802 family.

Switches can be used in order to reduce the number of competitors to the same shared medium in a network, by segmenting the overall network and bounding the frame transmission in a limited range. In a switched-network, users can receive and transmit at the same time by means of two separate channels, the so-called Full Duplex Switched Ethernet facilities that can be suitably used in Gigabit LANs.

Now, it is worth noting that:

- LLC functionalities are analogous to protocol adaptation functions that are used for the transportation of IP traffic over satellite devices;

- LEO payload with bridging/switching modules is envisioned for future satellite networking;

- Full-duplex techniques are consistent with satellite communication systems, where different channels are commonly adopted for uplink and downlink.

Thus, we firstly questioned how much existing Ethernet-like solutions could be reused to obtain a protocol harmonization for satellite and terrestrial devices; secondly, we investigated how existing mechanisms should be enhanced to mach with satellite-specific issues, and in particular with LEO mobility problems.

Our research turns in the exploitation of a cross-layer design of layers 2 and 3. In fact, layer 2 switching is performed on the basis of end-to-end connections to be established (known from the IP demand, which does not involve all the LEO network at once, but only a set of sub-networks, possibly separated), and by taking into account the knowledge of logical path changes 
due to the turnover of LEO satellite positions. In other words, we deploy virtual sub-networks (Virtual Local Area Networks, VLANs) that span that portion of the LEO network needed to accommodate the IP demand, while continuously adjusting the logical VLAN topology on the basis of the predicted satellites motion. As a result, it is possible to choose data-link connections in order to balance the network traffic, to optimize the usage of MAC resources and to differentiate users and services (i.e., groups to be handled in VLANs). In turn, an enhanced degree of connectivity, robustness and QoS is provided to the IP level.

\subsubsection{Protocol harmonization and implicit cross-layer design via IEEE VLAN}

Protocol harmonization is meant for QoS-aware internetworking of communication segments, avoiding intricacies due to satellite gateways needed to access a satellite sub-network, and also due to routing and path discovery to be managed for time-varying topologies, typical of non-GEO satellite fleets. As a consequence, a LEO satellite constellation could be merged at layer 2 with legacy terrestrial networks, and end-to-end communications could seamlessly use satellite and terrestrial links.

Cross-layer design of layers 2 and 3 is meant to avoid the negative impact of topological changes, deep signal shadowing, data loss, and reconfiguration of layer 2 links needed to span the entire network and to avoid loops. However, note that we deal here with implicit cross-layer design, since we aim at optimizing the resource management in the MAC layer to support IP QoS and robustness. The optimization of resource management is performed by joining the effectiveness of spanning tree algorithms and the possibility to configure remotely and proactively LEO switching devices on-the-fly. To this aim, a centralized path/VLAN manager is required that selects ISLs to be activated at any time instant, provides path redundancy by means of multiple disjointed VLANs, and avoids service discontinuity by switching the IP traffic from a VLAN to another.

The rationale of our proposal is based on the consideration that most of topology changes in the network can be foreseen from the knowledge of the satellite motion and from a statistical analysis of signal strength at receivers, so that the switched-network can be proactively managed.

In order to manage efficiently a switched-network, it is necessary to maintain only a sub-set of inter-node links to form a connected loop-free graph (i.e., a tree-like logical topology). This permits to confine broadcast traffic, eliminates looping frames, and, mostly important, makes easier the routing of data frames by exploiting a tree that connects all nodes without redundancy. However, the extraction of a tree from the original network graph has to be performed in accordance with traffic management criteria. Indeed, multiple trees could be adopted to segment the network traffic, to create virtual sub-networks, to perform load balancing, or also to provide some redundancy 
if a link abruptly fails. These advanced features are offered by IEEE standards for VLANs [41] that include LLC for switching and bridging, Spanning Tree Protocol (STP) and its variants Rapid STP (RSTP) [42] and Multiple STP (MSTP) [43], and VLAN tagging methods. IEEE VLAN and MSTP can be suitably adopted in order to simplify the management of a huge number of connections, even though adopting satellite switching implies the constitution of very large WANs or MANs where IP routing is unnecessary. Important advantages can be obtained, such as the possibility to exploit a particularly broad connectivity, or the possibility to eliminate IP route discovery latency, frequent inconsistencies in IP routing tables due to LEO topology changes, and path elaboration delays.

Although spanning tree protocols are able to rearrange their configuration after a link or a node fails, the satellite VLAN approach only works if spanning trees and VLANs are proactively adjusted when the LEO logical topology changes. Note that legacy reconfiguration procedures could take several seconds (due to the huge network diameter) during which the network graph results unconnected. However, the adoption of proactive mechanisms is reasonable since: $(i)$ the LEO satellite fleet is known a priori; (ii) the LEO fleet can be designed so that at lest one satellite is always visible in the target coverage area, and at least two satellites are visible during a handover event. Thus, the proactive management simply consists of setting up a new VLAN with a new spanning tree before the handover is performed, thus forcing a $V L A N$ handover before the physical handover. Note that, as for the VLAN handover procedure, it simply requires to change the tagging of frames at the edge of the satellite path from the old VLAN tag to the new one.

Considering the service offered to end-users, the adoption of VLANs with proactively managed multiple spanning trees allows avoiding: $(i)$ IP data-flow discontinuities due to physical topology changes, (ii) waste of large time intervals in spanning tree reconfigurations, triggered by the failure of a link or a node, and (iii) waste of bandwidth due to possible flooding effects after reconfigurations.

\subsubsection{Performance evaluation}

Multiple VLANs allow the network provider to hide network topology changes. In fact, during a topology transition, a new path will be available before the old path goes down. We include these different paths within different VLANs (i.e., addressed by different VLAN tags in the frame header) and switch to the new path during the topology transition. The VLAN manager knows the topology transition and enforces a VLAN tag change (i.e., a VLAN handover) at the edge of the network, so that each frame will follow a path in the VLAN identified by its new tag. In practice, we use multiple VLANs as redundant sub-networks.

Table 8.6 [44] reports what happens to frames generated in a message exchange between two terrestrial users at the edge of a Teledesic-like LEO 
network, when UDP is used, comparing the case in which a VLAN handover is adopted to a scenario without such a feature. We consider the case where only two users are in the network and only one connection is active $\left({ }^{2}\right)$. Summarizing, we can say that the absence of VLANs implies service discontinuities and long flooding phases after reconfigurations (due to the unidirectional nature of UDP exchange considered). Using VLANs, discontinuities of the service are avoided: packets are not filtered and end-to-end connectivity is not broken. The flooding after reconfiguration is bounded to the VLAN used after the handover; whereas, an STP-based approach would flood the entire network (STP uses a single tree for the entire network). Similar considerations can be made when TCP is used, with the exception of the occurrence of short flooding phases, due to frequent TCP ACKs.

\begin{tabular}{|c|c|c|}
\hline \multicolumn{3}{|c|}{ UDP } \\
\hline Event & Action (No VLAN) & Action (two VLANs) \\
\hline \multirow{2}{*}{$\begin{array}{l}\text { Service request } \\
\text { from Client }\end{array}$} & Flooding & Flooding in VLAN\#1 \\
\hline & $\begin{array}{c}\text { Switches learn Client } \\
\text { address }\end{array}$ & $\begin{array}{l}\text { VLAN\#1 switches learn } \\
\text { Client address }\end{array}$ \\
\hline \multirow{2}{*}{$\begin{array}{l}\text { Service response } \\
\text { from Server }\end{array}$} & Switching/no flooding & Switching/no flooding \\
\hline & $\begin{array}{c}\text { Switches learn Server } \\
\text { address }\end{array}$ & $\begin{array}{l}\text { VLAN\#1 switches learn } \\
\text { Server address }\end{array}$ \\
\hline Service data sent & Switched/no flooding & Switching/no flooding \\
\hline \multirow[t]{4}{*}{ Topology change } & Re-compute Spanning Tree & Handover to VLAN\#2 \\
\hline & $\begin{array}{l}\text { LAN temporarily } \\
\text { disconnected }\end{array}$ & $\begin{array}{l}\text { Re-compute CIST and } \\
\text { VLAN\#1 Spanning Tree }\end{array}$ \\
\hline & All frames discarded & $\begin{array}{l}\text { VLAN\#1 temporarily } \\
\text { disconnected }\end{array}$ \\
\hline & & $\begin{array}{c}\text { VLAN\#2 flooded, filtering } \\
\text { databases are empty }\end{array}$ \\
\hline $\begin{array}{c}\text { Downstream } \\
\text { (Upstream) frames } \\
\text { after } \\
\text { reconfiguration }\end{array}$ & \begin{tabular}{|} 
Network flooded by "new" \\
switches until an up- \\
(down-) stream frame is \\
sent by Client (Server)
\end{tabular} & $\begin{array}{c}\text { VLAN\#2 flooded by all } \\
\text { switches until an up- (down-) } \\
\text { stream frame is sent by Client } \\
\text { (Server) }\end{array}$ \\
\hline
\end{tabular}

Table 8.6: How topology changes affect UDP connections. Note that each network region that is managed by MSTP needs a Common Internal Spanning Tree (CIST) to interconnect all nodes in that region. See reference [44]. Copyright (C)2005 IEEE.

Table 8.7 [44] collects a set of actions performed by network entities at the occurrence of specific TCP-related events. The advantage of using proactive VLANs is clear from the comparison with the "legacy" behavior of switches,

2 This is the worst-case scenario, since switching devices need bidirectional flows in order to learn the route towards a remote user, otherwise frames are flooded in the VLAN. 
as described in the central column of the table, and the behavior of the VLAN with support for off-line reconfiguration, as described in the rightmost column: VLANs reduce flooding effects and off-line reconfiguration eliminates service discontinuities. However, a flooding phase is still needed in order to fill the filtering database (a layer-2 routing table built by bridges by monitoring incoming frames - source address and incoming port - to discover the outgoing port to be used to forward frames without the need of flooding) of the new VLAN.

\begin{tabular}{|c|c|c|}
\hline \multicolumn{3}{|c|}{ TCP } \\
\hline Event & Action (No VLAN) & Action (two VLANs) \\
\hline \multirow{2}{*}{$\begin{array}{c}\text { Request from } \\
\text { Client (TCP SYN) }\end{array}$} & Flooding & Flooding in VLAN\#1 \\
\hline & $\begin{array}{l}\text { Switches learn Client } \\
\text { address }\end{array}$ & $\begin{array}{c}\text { VLAN\#1 switches learn } \\
\text { Client address }\end{array}$ \\
\hline \multirow{2}{*}{$\begin{array}{c}\text { Response from } \\
\text { Server (TCP ACK) }\end{array}$} & Switching/no flooding & Switching/no flooding \\
\hline & $\begin{array}{c}\text { Switches learn Server } \\
\text { address }\end{array}$ & $\begin{array}{c}\text { VLAN\#1 switches learn } \\
\text { Server address }\end{array}$ \\
\hline$T C P S Y N-A C K$ & Switched/no flooding & Switched/no flooding \\
\hline Service data flow & Switched/no flooding & Switched/no flooding \\
\hline Client's $A C K$ & Switched/no flooding & Switched/no flooding \\
\hline \multirow[t]{3}{*}{ Topology changes } & Re-compute Spanning Tree & Switch to VLAN\#2 \\
\hline & $\begin{array}{l}\text { LAN temporarily } \\
\text { disconnected }\end{array}$ & $\begin{array}{c}\text { Re-compute CIST and } \\
\text { VLAN\#1 Spanning Tree }\end{array}$ \\
\hline & All frames discarded & $\begin{array}{l}\text { VLAN\#1 temporarily } \\
\text { disconnected }\end{array}$ \\
\hline $\begin{array}{c}\text { Downstream } \\
\text { (Upstream) frame } \\
\text { after } \\
\text { reconfiguration }\end{array}$ & $\begin{array}{c}\text { Flooded by "new" switches } \\
\text { until an up- (down-) } \\
\text { stream frame is sent by } \\
\text { Client (Server) }\end{array}$ & $\begin{array}{c}\text { Flooded in VLAN\#2 } \\
\text { switches until an up- (down-) } \\
\text { stream frame is sent by Client } \\
\text { (Server) }\end{array}$ \\
\hline
\end{tabular}

Table 8.7: How topology changes affect TCP connections. See reference [44]. Copyright (C)2005 IEEE.

In what follows, we show some results obtained in a scenario similar to the one depicted in Figure 8.17 [44], by means of the OPNET [45] simulator with modified bridging/switching devices. Satellite orbits are designed following the design principles of the Teledesic system, where LEO orbits have a 1375 $\mathrm{km}$ altitude and the satellite capacity is set to $32 \mathrm{Mbit} / \mathrm{s}$ for both uplink and downlink with terrestrial users, even though channels can be allotted to users as multiple of the $16 \mathrm{kbit} / \mathrm{s}$ basic channel. Two terrestrial bridges/switches are considered ( $\mathrm{T} 1$ and $\mathrm{T} 2$ in the figure). Users are located close to the terrestrial bridges; the longest path between two users in the simulation has a delay bounded to $200 \mathrm{~ms}$. We tested both UDP and TCP-based applications with and without VLAN supports.

Let us describe the generation of both UDP traffic and the TCP one. In 


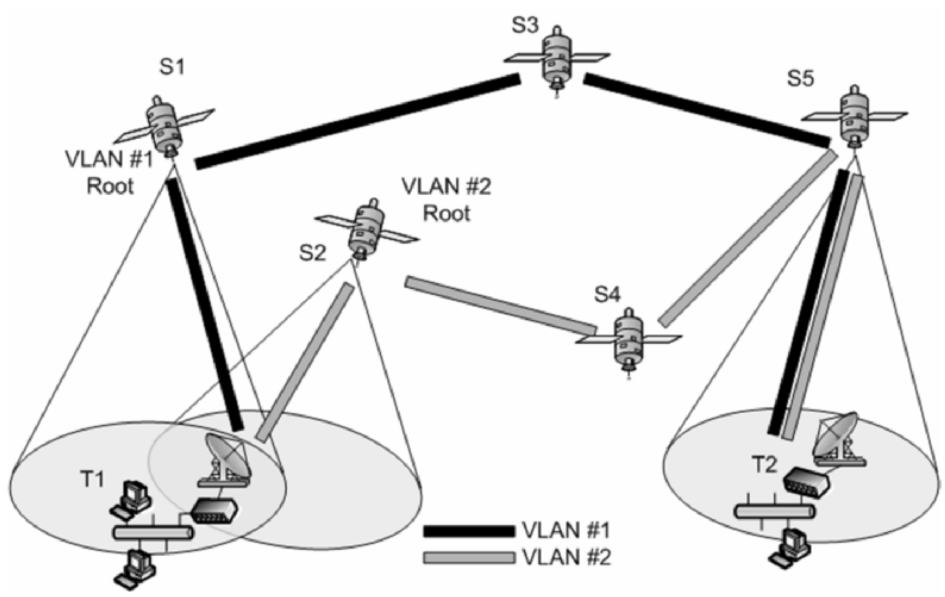

Fig. 8.17: Possible network topology with two VLANs available. See reference [44]. Copyright (C)2005 IEEE.

particular, UDP traffic is obtained via simulation of unidirectional constantrate video connections; also packet distribution is constant. Three UDP groups of users have been considered that differ in terms of both bit-rate (i.e., 256, 128 and $64 \mathrm{kbit} / \mathrm{s}$, respectively for Class I, II and III users), and the average request inter-arrival time (which is exponentially distributed, so that the arrival process of connection requests is Poisson). Mean inter-arrival times are, respectively: $45 \mathrm{~s}$ for Class I, $22.5 \mathrm{~s}$ for Class II, and $11.25 \mathrm{~s}$ for Class III. Each connection has a duration exponentially distributed with mean value of $180 \mathrm{~s}$. The number of users in a group is selected so that each UDP group offers $1 \mathrm{Mbit} / \mathrm{s}$ traffic in average, directed from nodes located in T1 to T2.

As for TCP-based traffic, the following results have been obtained by considering the separate contribution of three groups of FTP users. Every user, located in T1, requests files of $B$ bytes, where $B$ is exponentially distributed with a mean of 5,000,000 bytes, while the file request inter-arrival time is exponentially distributed with a mean of $5 \mathrm{~s}$. User groups are differentiated based on the available resources allotted in the access link: Class I (High Rate) has an aggregate guaranteed rate of $512 \mathrm{kbit} / \mathrm{s}$ for the downstream and $128 \mathrm{kbit} / \mathrm{s}$ for the upstream; Class II (Medium Rate) has an aggregate guaranteed rate of $128 \mathrm{kbit} / \mathrm{s}$ for the downstream and $32 \mathrm{kbit} / \mathrm{s}$ for the upstream; eventually, Class III (Low Rate) has an aggregate guaranteed rate of $64 \mathrm{kbit} / \mathrm{s}$ for the downstream and $16 \mathrm{kbit} / \mathrm{s}$ for the upstream. Each group saturates its link capacity due the high file request rate $(5$ files per second are requested, i.e., about 25 MByte per second, which requires at least 120 Mbit/s plus the protocol overhead: the system is overloaded and the number of FTP requests overwhelms the number of FTP sessions that reach the end of transmission). As a matter of fact, simulations confirm the behavior described 
in Table 8.6 for UDP and the considerations about TCP in Table 8.7. Details are provided below.

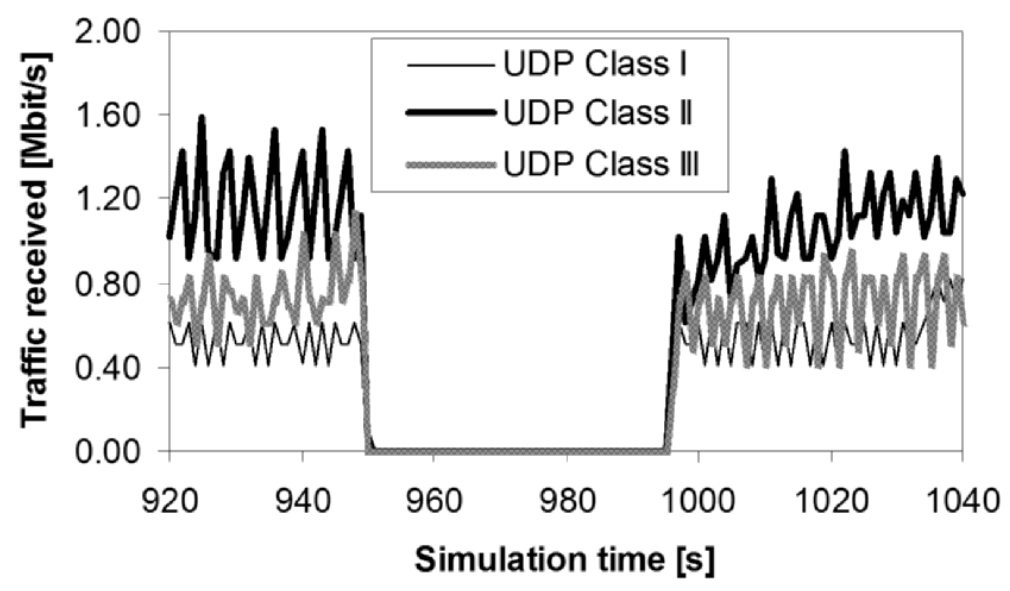

Fig. 8.18: UDP throughput with STP, no VLANs.

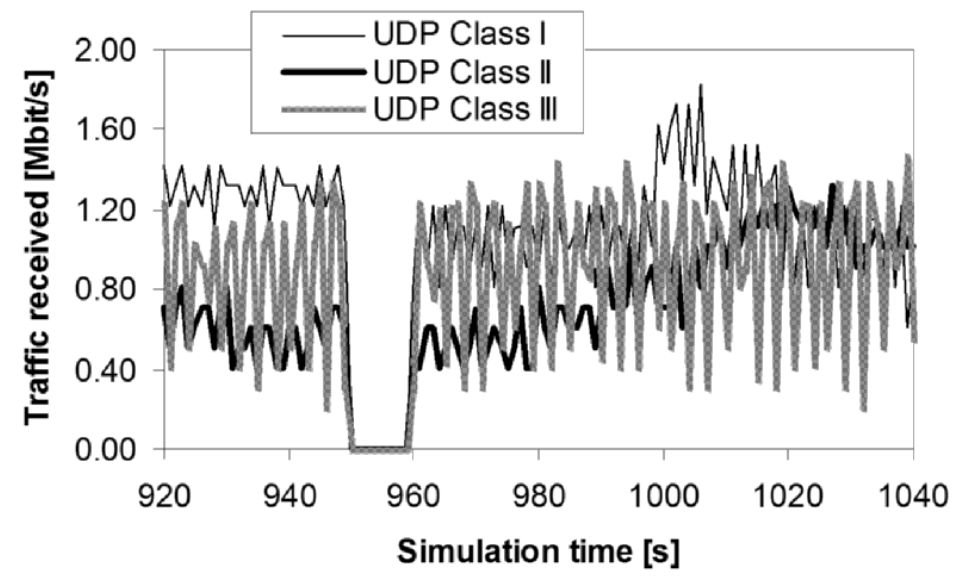

Fig. 8.19: UDP throughput with RTSP, no VLANs.

Figures 8.18 to 8.20 show the throughput of a unidirectional UDP connection between two remote hosts. In the simulations, a physical topology change occurred at $t=950 \mathrm{~s}$, and one can notice that a traditional STP approach requires up to $45 \mathrm{~s}$ to recover the path; using RSTP this time is shortened, 
but several seconds, about $10 \mathrm{~s}$, are still needed to reconfigure the large switched-network. On the contrary, preconfigured VLANs allow a seamless handover, without service discontinuities. In Figure 8.20, a VLAN handover is enforced at $t=940 \mathrm{~s}$, just a few seconds before the physical topology change. Similar considerations could be made by considering bidirectional UDP flows, where the traffic is generated in each direction as in the unidirectional case.

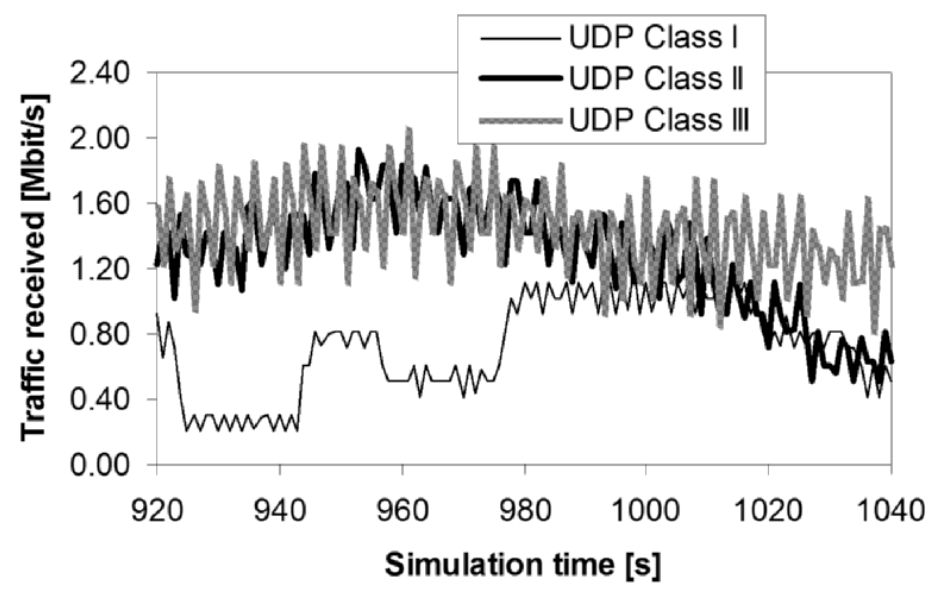

Fig. 8.20: UDP throughput with VLAN handover.

Figures 8.21 and 8.22 depict the throughput of a TCP connection for hosts requesting FTP files from a network server. In this case, traffic flows are bidirectional, due to the presence of ACK packets in the return channel, even though the connection is strongly asymmetric. In these simulations, a topology change occurred at $t=800 \mathrm{~s}$. By using STP (Figure 8.21) or RSTP, we can notice a service interruption with a duration similar to that experienced in UDP simulations, but the effect is partially masked by the build up of long queues at the last satellite-to-ground station link, especially for the TCP Class III, which is allotted the minimum resources. It is worth noting that after the network reconfiguration, each traffic group aggregate suffers from high fluctuation due to the synchronization of TCP flows after the outage period. In particular, Class I experiences a very drastic fluctuation, while lower rate traffic classes grow very slowly. Eventually, if we consider the adoption of VLAN (Figure 8.22), with a handover operated at $t=790 \mathrm{~s}$, no significant variation can be noted in the traffic aggregate of each class. Again, off-line configured VLANs allow ground stations to switch seamlessly between VLANs, and avoid service discontinuities.

As for the flooding effects due to topology changes, first we consider unidirectional UDP flows in the network, from site T1 to site T2. Figures 


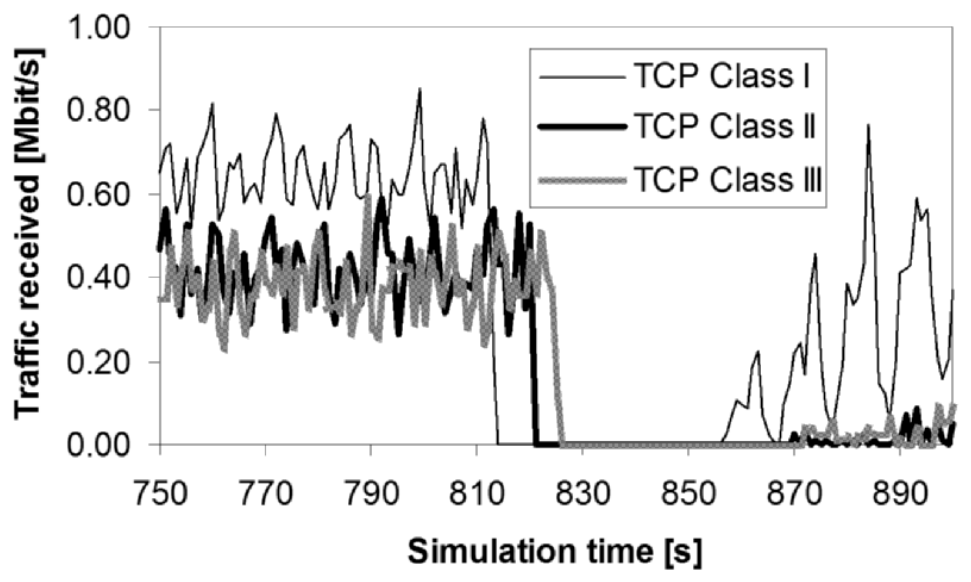

Fig. 8.21: TCP throughput with STP, no VLANs.

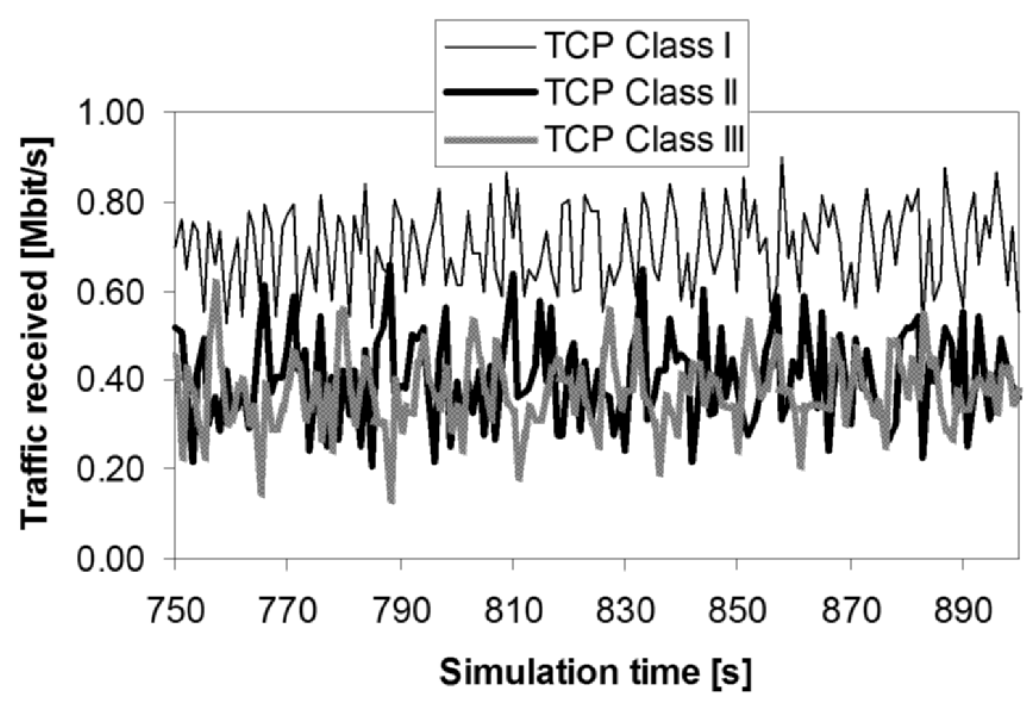

Fig. 8.22: TCP throughput with VLAN handover.

8.23 and 8.24 represent data flooded by switches when no appropriate entries are found in the filtering database. Each flooded data frame is accounted for only once, no matter if multiple switches will flow again the same frame. In practice, a flooding phase starts after an automatic route change, performed by RSTP (or STP, not showed here). This is the reason why Figure 8.23 shows flooded packets for multiple sources after the first disrupted path is recovered, which is not mandatory for the data path we are interested to. 


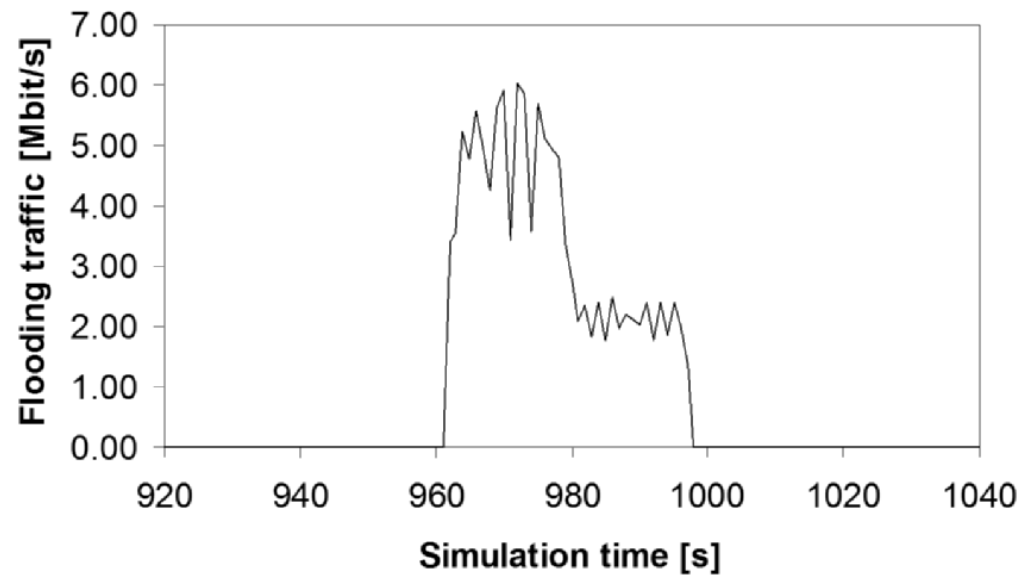

Fig. 8.23: Unidirectional UDP connections: normalized aggregated flooding (RSTP).

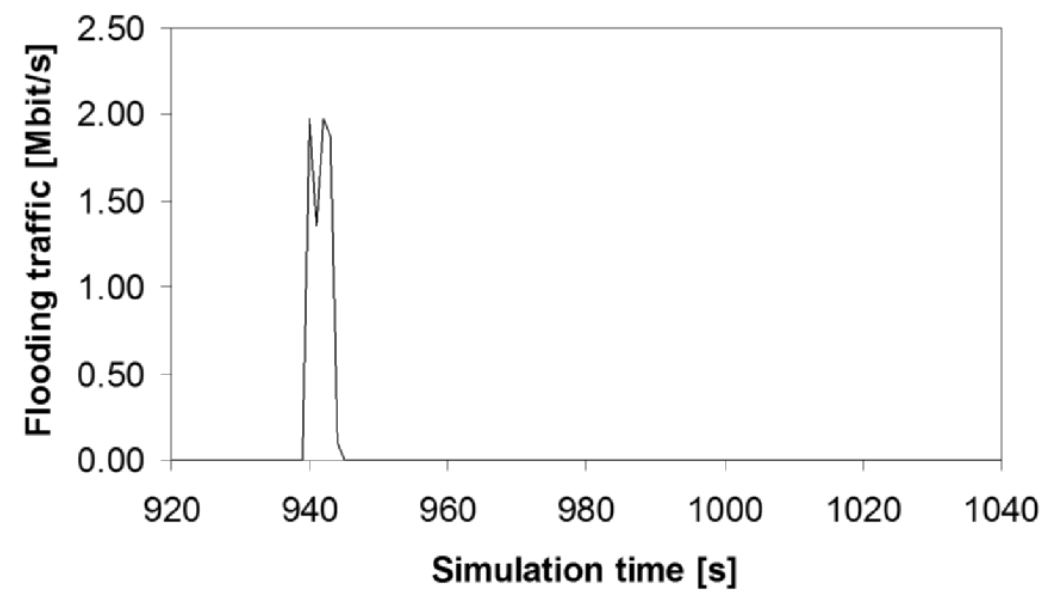

Fig. 8.24: Unidirectional UDP connections: normalized aggregated flooding (VLAN).

Thus, the flooding phase ends only after the network is fully reconfigured and a new frame is sent in the reverse path for each user (i.e., after a new request is sent per each UDP traffic class, which is represented, in these simulations, by a single user). Figure 8.24 shows that by adopting VLAN-based network management, a simple VLAN handover is required a few seconds before the original path goes down. However, VLAN handover requires a brief flooding phase just after the handover, since the filtering database learning phase has to be performed as well. 
As for the flooding effects in case of bidirectional UDP connections, in the same network and traffic conditions as before, it can be found a very limited flooding due to the fact that an intense traffic is used in both directions, so that database learning phases are very short.

Figure 8.25 shows the burden of flooding data for TCP connections when RSTP is adopted. Data are related to frames carrying TCP segments (data and ACKs) that experience at least one duplication in a generic network node. Due to the asymmetric nature of TCP upstream and downstream (i.e., the different size and bandwidth occupied by data and ACKs), it is appropriate to distinguish between the amount of flooded bits and the number of flooded frames: we represent the flooding in terms of flooded packets, which give a normalized estimate of how much dangerous the flooding can be. Note that flooding occurs while the network is reconfiguring itself. However, RSTP operation allows data to be flooded immediately after the link failure. In fact, when using spanning trees, each link is represented as an arch of an oriented graph. The orientation of each arch is from the root to the leaves of the tree. Thus, a link connects an up-node to a down-node. Using RSTP, the down-node is in charge of sensing the link failure and starting the recovery phase; the down-node is also allowed to use alternative links to reach the root of the tree. The most important cause of flooding in RSTP is given by frames that reach a down-node of a broken link. Eventually, flooding is almost completely avoided by using VLANs, as stated by Figure 8.26.

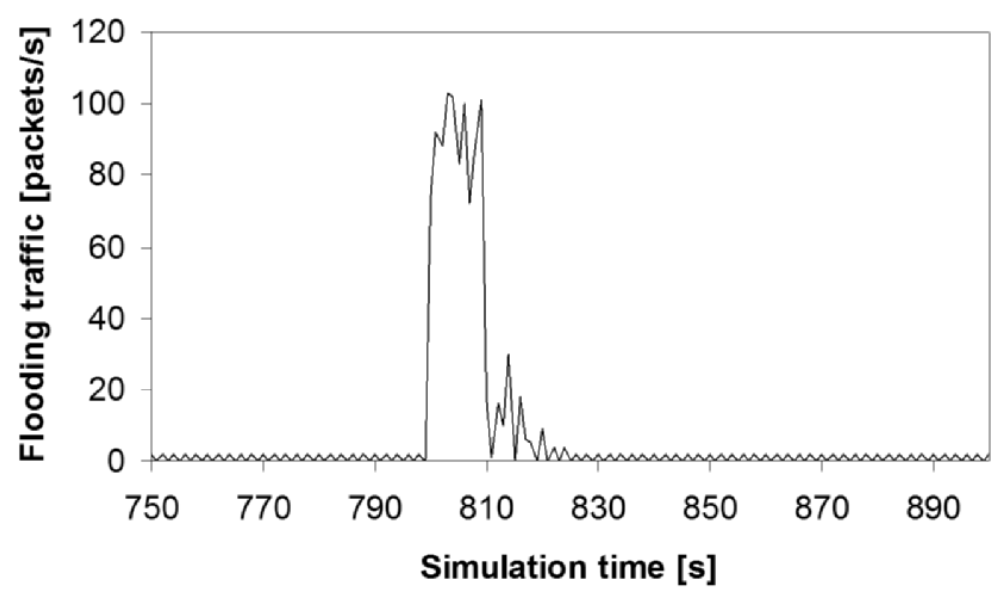

Fig. 8.25: TCP connections: normalized aggregated flooding (RSTP). 


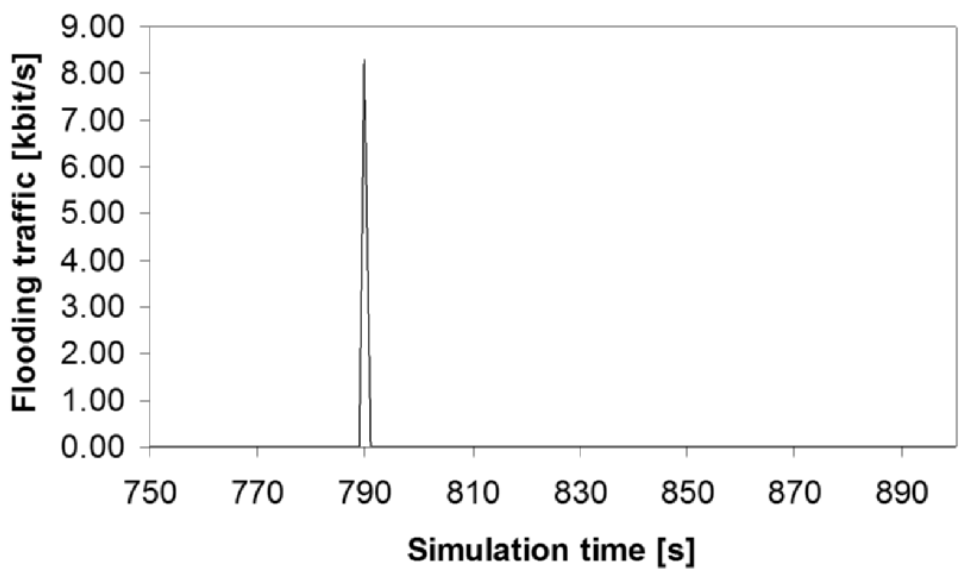

Fig. 8.26: TCP connections: normalized aggregated flooding (VLAN).

\subsection{Conclusions}

Over the past decades, with the emergence of many multimedia Internet applications and services, the research community has devoted a big effort in an attempt to satisfy their stringent and varied QoS requirements. A clear example of this effort is the initiative by IETF in proposing two IP QoS frameworks. These frameworks are mainly designed with terrestrial networks in mind. However, the problems of achieving QoS in networks with wireless medium such as satellite networks are much more complicated since the link is dependent on channel conditions. Hence, the resource management block is vital in realizing the IP QoS frameworks.

Standard mechanisms which operate solely in the network layer most often cannot guarantee the QoS when the end-to-end path involves satellite or wireless networks as they disregard the variability of channel conditions. This leads to the investigation of utilizing MAC layer resource management schemes or protocols to improve this situation. More recently, the idea of using cross-layer techniques further open up the potential of what can be achieved in terms of QoS provision.

Being in adjacent layers in the protocol stack, resource management (layer 2 ) in satellite networks is always tightly coupled with the IP QoS frameworks (layer 3). This Chapter has been dedicated to the cross-layer interactions and issues between these two layers. A review of the current state of the IP QoS frameworks in relation with the satellite network shows that DiffServ is being increasingly accepted and an example implementation of relative DiffServ is given as an illustration on how MAC layer scheduling can support the QoS provisioning. The problem of mapping between the QoS mechanisms operating at the two layers has been formulated and a measurement-based approach has 
been presented. The problem is also discussed in two other scenarios; namely the dual network access and Switched Ethernet over LEO satellites.

From the discussions and results presented in this Chapter, it is clear that achieving IP QoS in a satellite environment can certainly benefit from cross-layer mechanisms from layer 2. Nevertheless, caution must be observed when designing such cross-layer schemes. Uncontrolled implementation of cross-layer mechanisms may cause other problems that may not be apparent in a short period of time. Cross-layer design aimed at improving a specific performance metric may not have the entire system performance considered while cross-layer design involving multiple layers may lead to 'spaghetti design' with high number of convoluted interactions. All these aspects will increase system complexity and hence will pose problems for future innovations. Worse, system update may require complete redesign. Another example of a negative impact of uncontrolled cross-layer design is on network security issues: the increased interactions among layers may increase the channels for security attacks. In conclusion, designers must have the long-term effects in mind. 


\section{References}

[1] S. Shenker, J. Wroclawski, "General Characterization Parameters for Integrated Service Network Elements", IETF RFC 2215, September 1997.

[2] R. Braden, D. Clark, S. Shenker, "Integrated Services in the Internet Architecture: an Overview", IETF RFC 1633, June 1994.

[3] R. Braden, L. Zhang, S. Berson, S. Herzog, S. Jamin, "Resource Reservation Protocol (RSVP) - Version 1 Functional Specification", IETF RFC 2205, September 1997.

[4] S. Shenker, C. Partridge, R. Guerin, "Specification of Guaranteed Quality of Service", IETF RFC 2212, September 1997.

[5] J. Wroclawski, "Specification of the Controlled-Load Network Element Service", IETF RFC 2211, September 1997.

[6] S. Blake, D. Black, M. Carlson, E. Davies, Z. Wang, W. Weiss, "An Architecture for Differentiated Services", IETF RFC 2475, December 1998.

[7] C. Dovrolis, D. Stiliadis, P. Ramanathan, "Proportional Differentiated Services: Delay Differentiation and Packet Scheduling", IEEE/ACM Transactions on Networking, Vol. 10, No. 1, pp. 12-26, February 2002.

[8] K. Wang. Quality of Service Assurances in Multihop Wireless Networks. PhD. Dissertation, University of Wisconsin-Madison, 2003.

[9] Y. Xue, K. Chen, K. Nahrstedt, "Achieving Proportional Delay Differentiation in Wireless LAN via Cross-Layer Scheduling", Journal of Wireless Communications \& Mobile Computing, Vol. 4, No. 8, pp. 849-866, November 2004.

[10] W. K. Chai, M. Karaliopoulos, G. Pavlou, "Scheduling for Proportional Differentiated Service Provision in Geostationary Bandwidth on Demand Satellite Networks", in Proc. of IEEE GLOBECOM 2005, St. Louis, MO, USA, November 28 - December 2, 2005.

[11] E. Rosen, A. Viswanathan, R. Callon, "Multiprotocol Label Switching Architecture", IETF RFC 3031, January 2001.

[12] ETSI, "Digital Video Broadcasting (DVB); Interaction channel for satellite distribution systems," ETSI European Standard (Telecommunications series), EN 301790 V1.3.1 (2003-03).

[13] G. Açar. End-To-End Resource Management in Geostationary Satellite Networks. PhD. Dissertation, University of London, November 2001.

[14] L. Kleinrock. Queueing Systems. New York, Wiley, 1976, Vol. II. 
[15] ETSI, "Satellite Earth Stations and Systems (SES). Broadband Satellite Multimedia, Services and Architectures", ETSI Technical Report, TR 101984 V1.1.1, November 2002.

[16] ETSI, "Satellite Earth Stations and Systems (SES). Broadband Satellite Multimedia, IP over Satellite", ETSI Technical Report, TR 101985 V1.1.2, November 2002.

[17] N. Iuoras, T. Le-Ngoc, "Dynamic Capacity Allocation for Quality-Of-Service Support in IP-Based Satellite Networks", IEEE Wireless Communications Magazine, Vol. 12, No. 5, pp. 14-20, October 2005.

[18] N. Iuoras, T. Le-Ngoc, M. Ashour, T. Elshabrawy, "An IP-Based Satellite Communication System Architecture for Interactive Multimedia Services", International Journal of Satellite Communications and Networking, Vol. 21, No. 4-5, pp. 401-426, July-October 2003.

[19] T. Le-Ngoc, V. Leung, P. Takats, P. Garland, "Interactive Multimedia Satellite Access Communications", IEEE Communications Magazine, Vol. 41, No. 7, pp. 78-85, July 2003.

[20] S. Combes, L. Goegebeur, M. Fitch, G. Hernandez, A. Iuoras, S. Pirio, "Integrated Resources and QoS Management in DVB-RCS Networks", in Proc. of the $22^{\text {nd }}$ AIAA International Communications Satellite Systems Conference \&5 Exhibit 2004 (ICSSC), Monterey, CA, May 2004.

[21] M. Marchese, M. Mongelli, "Rate Control Optimization for Bandwidth Provision over Satellite Independent Service Access Points", in Proc. of IEEE Globecom 2005, St. Louis, MO, USA, pp. 3237-3241, November 28 - December $2,2005$.

[22] M. Marchese, M. Mongelli, "On-Line Bandwidth Control for Quality of Service Mapping over Satellite Independent Service Access Points", Computer Networks, Vol. 50, No. 12, pp. 1885-2126, August 2006.

[23] M. Marchese, M. Mongelli, "Real-Time Bandwidth Control for QoS Mapping of Loss and Delay Constraints over Satellite Independent Service Access Points", submitted to IEEE Transactions on Wireless Communications.

[24] C. G. Cassandras, G. Sun, C. G. Panayiotou, Y. Wardi, "Perturbation Analysis and Control of Two-Class Stochastic Fluid Models for Communication Networks", IEEE Transactions on Automatic Control, Vol. 48, No. 5, pp. 770-782, May 2003.

[25] N. Celandroni, F. Davoli, E. Ferro, "Static and Dynamic Resource Allocation in a Multiservice Satellite Network with Fading", International Journal of Satellite Communications and Networking, Vol. 21, No. 4-5, pp. 469-487, July-October 2003.

[26] Y. Wardi, B. Melamed, C. G. Cassandras, C. G. Panayiotou, "Online IPA Gradient Estimators in Stochastic Continuous Fluid Models", Journal of Optimization Theory and Applications, Vol. 115, No. 2, pp. 369-405, November 2002.

[27] R. Guérin, H. Ahmadi, M. Naghshineh, "Equivalent Capacity and its Application to Bandwidth Allocation in High-Speed Networks", IEEE Journal on Selected Areas in Communications, Vol. 9, No. 7, pp. 968-981, September 1991.

[28] Web site with URL:

http://www-tkn.ee.tu-berlin.de/research/trace/trace.html. 
[29] L. Rizzo, L. Vicisano, "RMDP: an FEC-Based Reliable Multicast Protocol for Wireless Environments", Mobile Computer and Communication Review, Vol. 2, No. 2, pp. 23-31, April 1998.

[30] M. Zorzi, "Performance of FEC and ARQ Error Control in Bursty Under Delay Constraints", in Proc. of VTC '98, Ottawa, Canada, May 1998.

[31] P. Barsocchi, A. Gotta, F. Potortì, F. J. González-Castaño, F. Gil-Castiñeira, J. I. Moreno, A. Cuevas, "Experimental Results with Forward Erasure Correction and Real Video Streaming in Hybrid Wireless Networks", in Proc. of IEEE International Symposium on Wireless Communication Systems 2005 (ISWCS 2005), ISBN 0-7803-9206-X, Siena, Italy, September 5-9, 2005.

[32] E. Feltrin, E. Weller, E. Martin, K. Zamani, "Implementation of a Satellite Network Based on Skyplex Technology in Ka Band", in Proc. of $9^{\text {th }} \mathrm{Ka}$ and Broadband Communications Conference, Ischia, Italy, November 2003.

[33] Web site with URL: http://votos.isti.cnr.it.

[34] L. Rizzo, "Effective Erasure Codes for Reliable Computer Communication Protocols", ACM Computer Communication Review, Vol. 27, No. 2, pp. 24-36, April 1997.

[35] ANSI, "American National Standard for Telecommunications - Digital Transport of One-Way Video Signals - Parameters for Objective Performance Assessment", T1.801.03, 1996.

[36] ATIS, "Objective Video Quality Measurement Using a Peak Signal-to-Noise Ratio (PSNR) Full Reference Technique", Alliance for Telecommunications Industry Solutions, 1200 G Street, NW, Suite 500, Washington, DC 20005, Technical Report T1.TR.74, October 2001.

[37] A. Cuevas et al. "Usability and Evaluation of a Deployed 4G Network Prototype", Journal of Communications and Networks (ISSN: 1229-2370), Vol. 7, No. 2, pp. 222-230, June 2005.

[38] M. Liebsch et al., "Candidate Access Router Discovery (CARD)", IETF RFC 4066, July 2005.

[39] ANSI/IEEE Std 802.2, 1998 Edition, "Part2: Logical Link Control".

[40] ANSI/IEEE Std 802.1D, 1998 Edition, "Part 3: Media Access Control (MAC) Bridges".

[41] IEEE, "802.1Q - IEEE Standards for Local and Metropolitan Area Networks Virtual Bridged Local Area Networks", Std 802.1QT, 2003.

[42] IEEE, "IEEE Standard for Local and Metropolitan Area Networks - Common specifications Part 3: Media Access Control (MAC) Bridges - Amendment 2: Rapid Reconfiguration", Std 802.1w-2001.

[43] IEEE, "802.1s - IEEE Standards for Local and Metropolitan Area Networks - Virtual Bridged Local Area Networks - Amendment 3: Multiple Spanning Trees", Std 802.1sT-2002.

[44] V. Mancuso, G. Bianchi, N. Blefari Melazzi, U. Birnbacher, "Switched Ethernet Networking over LEO Satellite", in Proc. of IEEE International Symposium on Wireless Communication Systems 2005 (ISWCS 2005), ISBN 0-7803-9206-X, Siena, Italy, September 5-9, 2005.

[45] The official OPNET Internet site with URL: http://www.opnet.com. 